\title{
Theranostic multimodular potential of zinc-doped ferrite-saturated metal-binding protein-loaded novel nanocapsules in cancers
}

This article was published in the following Dove Press journal:

International Journal of Nanomedicine

I April 2016

Number of times this article has been viewed

\author{
Sishir K Kamalapuram' \\ Rupinder K Kanwar' \\ Kislay Roy' \\ Rajneesh Chaudhary' \\ Rakesh Sehgal ${ }^{2}$ \\ Jagat R Kanwar' \\ 'Nanomedicine Laboratory of \\ Immunology and Molecular \\ Biomedical Research, School of \\ Medicine, Centre for Molecular and \\ Medical Research, Faculty of Health, \\ Deakin University, Geelong, VIC, \\ Australia; ${ }^{2}$ Department of Medical \\ Parasitology, Postgraduate Institute \\ of Medical Education and Research, \\ Chandigarh, India
}

\begin{abstract}
The present study successfully developed orally deliverable multimodular zinc ( $\mathrm{Zn}$ ) iron oxide $\left(\mathrm{Fe}_{3} \mathrm{O}_{4}\right)$-saturated bovine lactoferrin (bLf)-loaded polymeric nanocapsules (NCs), and evaluated their theranostic potential (antitumor efficacy, magnetophotothermal efficacy and imaging capability) in an in vivo human xenograft $\mathrm{CpG}$-island methylator phenotype (CIMP)- $1^{+} /$CIMP2-/chromosome instability-positive colonic adenocarcinoma (Caco2) and claudin-low, triple-negative (ER-/PR-/HER2-; MDA-MB-231) breast cancer model. Mice fed orally on the $\mathrm{Zn}$-Fe-bLf NC diet showed downregulation in tumor volume and complete regression in tumor volume after 45 days of feeding. In human xenograft colon cancer, vehicle-control $\mathrm{NC}$ diet-group $(\mathrm{n}=5)$ mice showed a tumor volume of $52.28 \pm 11.55 \mathrm{~mm}^{3}$, and $\mathrm{Zn}-\mathrm{Fe}-\mathrm{bLf} \mathrm{NC}$ diet $(\mathrm{n}=5)$-treated mice had a tumor-volume of $0.10 \pm 0.073 \mathrm{~mm}^{3}$. In the human xenograft breast cancer model, Zn-Fe-bLf NC diet ( $\mathrm{n}=5)$-treated mice showed a tumor volume of $0.051 \pm 0.062 \mathrm{~mm}^{3}$ within 40 days of feeding. Live mouse imaging conducted by near-infrared fluorescence imaging of Zn-Fe-bLf NCs showed tumor site-specific localization and regression of colon and breast tumor volume. Ex vivo fluorescence-imaging analysis of the vital organs of mice exhibited sparse localization patterns of Zn-Fe-bLf NCs and also confirmed tumor-specific selective localization patterns of Zn-Fe-bLf NCs. Dual imaging using magnetic resonance imaging and computerized tomography scans revealed an unprecedented theranostic ability of the Zn-Fe-bLf NCs. These observations warrant consideration of multimodular Zn-Fe-bLf NCs for real-time cancer imaging and simultaneous cancer-targeted therapy.
\end{abstract}

Keywords: theranostic, multimodular cancer therapy, nanocapsules, nanomedicine, real-time imaging

\section{Introduction}

Therapeutics derived from natural products are safe, highly biocompatible, and exhibit negligible toxicity. Due to these characteristics, cancer researchers are in search of novel noble therapeutic compounds as nanotheranostic platforms for cancer. ${ }^{1}$ Cancer has evolved as an increasingly complex disease, due to the innumerable cancer-growth mechanisms escaping the normal growth patterns that lead to severe disease states. ${ }^{2}$ Due to these growth trends, it is very difficult for conventional chemotherapeutic drugs and traditional therapeutic platforms to achieve effective treatment of cancers..$^{3-5}$ Iron-saturated bovine lactoferrin (Fe-bLf) has been extensively studied for its prominent ability in helping the immune system to combat cancer and also in generation of immune cells, such as B lymphocytes, increasing antitumor macrophage activity, and increased production of wide range of interleukins. ${ }^{3,6-9} \mathrm{Fe}-\mathrm{bLf}$-derived nanotherapeutics exhibit apoptotic signaling mechanisms, and further Fe-bLf had a crucial role in the
Correspondence: Jagat R Kanwar Nanomedicine Laboratory of Immunology and Molecular Biology, School of Medicine, Centre for Molecular and Medical Research, Faculty of Health School of Medicine, Deakin University, 75 Pigdons Road, Waurn Ponds, Geelong, VIC 3217, Australia

Tel +6 I 35227 II 48

Fax +6I 352272539

Email jagat.kanwar@deakin.edu.au (c) (1) (5) 2016 Kamalapuram et al. This work is published and licensed by Dove Medical Press Limited. The full terms of this license are available at https://www.dovepress.com/terms.php (c) hereby accept the Terms. Non-commercial uses of the work are permitted without any further permission from Dove Medical Press Limited, provided the work is properly attributed. For permission for commercial use of this work, please see paragraphs 4.2 and 5 of our Terms (https://www.dovepress.com/terms.php). 
modulation of key stem cell-signaling mechanisms, including inhibiting survivin expression and EpCAM signaling, and other noteworthy observations included downregulation of the prominent stem cell markers CD44, CD133, and CD166. ${ }^{8}$ Interestingly, Fe-bLf nanocarriers/nanocapsules (NCs) exploited several internalization receptors, such as LRP1 and -2, TfRs, and DMT1 iron-metabolism receptors. ${ }^{8,10}$

Cancer nanotheranostics is an ultramodern field that offers mouthwatering approaches in effective targeting of cancer by a three-way integrated approach: diagnostic, therapeutic, and monitoring of the disease condition. Due to its lucrative benefits, cancer nanotheranostic approaches and preferably eco-friendly natural product-derived compounds are in the limelight of research. ${ }^{11}$ In consideration of the importance of the multimodular nanotheranostic drug-delivery systems for cancer treatment, in terms of selective timely drug delivery at the tumor site and also real-time monitoring of disease conditions and cancer therapy, ${ }^{12}$ there is an urgent need for such agents in cancer diagnosis and therapy.

The strategy used to obtain maximum possible magnetization with a considerably reduced toxicity with less contrast agent consists of doping the ferrite-nanoparticle core of the employed nanocomplex. In order to achieve this goal, the spinel structure of the ferrite-nanoparticle core can be doped with metallic cations, such as copper, cobalt, manganese, and zinc, in order to enhance their effective saturation-magnetization value. The US Food and Drug Administration has published a daily value for intake of the micronutrients zinc and iron to be 15 and $18 \mathrm{mg}$, respectively, considerably higher than the daily value for manganese and cobalt at $2 \mathrm{mg} .{ }^{13}$ Moreover, replacement of iron with zinc will additionally reduce the chances of iron overloading by replacing ferrous ions with zinc cations. Therefore, using zinc as a dopant is a much safer approach to enhance the saturation-magnetization value of ferrite nanoparticles than manganese, copper, or nickel.

The present study is the first attempt to develop multimodular $\mathrm{Zn}$-doped iron oxide $\left(\mathrm{Fe}_{3} \mathrm{O}_{4}\right)$-saturated bLf-loaded polymeric NCs (Zn-Fe-bLf NCs) for theranostic application in cancer therapy and imaging. Research conducted on zinc absorption has shown an increased correlation of anticancer activity with respect to zinc levels in the body. Therefore, increased zinc levels help in inhibiting cancer growthsignaling mechanisms. ${ }^{14}$ Further, zinc as a micronutrient enhances immune-system activity in retaliation against tumor growth. Zinc has been classified as an important element for the human body as a micronutrient, and also helps in immunosignaling mechanisms, enriching the body's ability to fight against diseases. ${ }^{14}$ Therefore, we utilized a Zn-Fe-bLf nanoformulation for examining its anticancer ability and also imaging ability in vivo.

Microsatellite-stable (MSS), chromosome instability $(\mathrm{CIN})^{+}, \mathrm{CpG}$-island methylator phenotype (CIMP)- $1^{+}$/ CIMP2-/ ${ }^{-} \mathrm{IN}^{+}$colonic adenocarcinoma ${ }^{15-17}$ and claudin-low, triple-negative $\left(\mathrm{ER}^{-} / \mathrm{PR}^{-} / \mathrm{HER}^{-}\right)$breast carcinoma ${ }^{10}$ are the most widespread cancers expressing specialized molecular characteristics, which enable them to promote tumor progression and therapeutic resistance. ${ }^{18-20}$ Current therapeutic approaches include the use of inhibitors for triple-negative breast cancer: P7170 (synthetic inhibitor), PP2A-B55 $\beta$, IL15RA for $\mathrm{CIMP}^{+} / \mathrm{CIMP}^{-} / \mathrm{CIN}^{+}$colonic adenocarcinoma, and the DNA methyltransferase inhibitor 5-azacitidine and the histone deacetylase inhibitor AR-42. ${ }^{15-17}$

In the present study, Zn-Fe-bLf NCs showed enhanced anticancer efficacy in the in vivo human xenograft $\mathrm{CIMP}^{+}$ CIMP2- $\mathrm{CIN}^{+}$colonic adenocarcinoma and claudin-low, triple-negative breast cancer model and near-infrared (NIR) fluorescence imaging enabled real-time monitoring of drug localization at the tumor site. The multimodular imaging capability employing dual-mode imaging strategies, such as magnetic resonance imaging (MRI) and computed tomography (CT), imparted to these NCs by the crucial zinc- and iron-based composition provides it an upper hand when compared to other currently available theranostic agents. Therefore, multimodular Zn-Fe-bLf NCs warrant future improvised extensive in vivo studies and clinical studies toward the development of effective nanotheranostic systems for cancer treatment. ${ }^{12}$

\section{Materials and methods}

$\mathrm{CIN}^{+}$, microsatellite-stable, CIMP1+, CIMP2- colon cancer (Caco2) and claudin-low, triple-negative $\left(\mathrm{ER}^{-} / \mathrm{PR}^{-} / \mathrm{HER}^{-}\right)$ breast cancer (MDA-MB-231) cell lines were purchased from the American Type Culture Collection (Manassas, VA, USA). Chitosan (20-200 cP, low molecular weight), sodium triphosphate, $0.25 \%$ trypsin-ethylenediaminetetraacetic acid (EDTA), penicillin-streptomycin, sodium alginate, and calcium chloride were obtained from Sigma-Aldrich Co (St Louis, MO, USA). Human cancer cell-culture essentials were purchased from GE Healthcare and BD Biosciences. A VivoTag XL fluorescent tag was obtained from PerkinElmer Inc (Waltham, MA, USA). Vector mounting media and a horseradish peroxidase-conjugate kit were purchased from Vector Laboratories, (Burlingame, CA, USA). Crystal violet stain was obtained from Thermo Fisher Scientific (Waltham, MA, USA). 


\section{Fabrication of alginate-coated chitosan nanogel-encapsulated zinc $\mathrm{Fe}_{3} \mathrm{O}_{4}$ - saturated bovine lactoferrin nanocapsules}

The fabrication of Zn-Fe-bLf NCs was carried out according to previous methods with slight modifications. ${ }^{7,8,12}$ Briefly, a 1:3 ratio of the $250 \mathrm{mM}$ zinc ferrite $\left(\mathrm{Zn}_{x} \mathrm{Fe}_{3-\mathrm{x}} \mathrm{O}_{4}\right)$ and $600 \mathrm{mM}$ of nitrilotriacetic acid (NTA) disodium salt (molecular weight 235.10) were dissolved in sterile water. Further, $\mathrm{pH}$ was adjusted to 7 . Later, $0.1 \mathrm{M}$ solid sodium bicarbonate was added to $0.1 \%$ solution of apo-bLf and $\mathrm{pH}$ adjusted to 8 . Then, the freshly prepared Zn-Fe-NTA solution was added dropwise to prepared apo-bLf solution while magnetically stirring the solution. In the presence of carbonate ions, structural confirmation of the Lf molecule possessing iron-binding pockets initiates the uptake of iron molecules.

The Zn-Fe-NTA solution was added to the protein solution until a reddish black-brown color solution was formed. This color formation signifies the $100 \%$ iron saturation of Lf. Further, the obtained mixture was subjected to overnight magnetic stirring at $4^{\circ} \mathrm{C}$ in order to stabilize the Zn-Fe-bLf complex. Later on, the Zn-Fe-bLf solution was separated by centrifugation techniques at 5,000 rpm for 10 minutes. The obtained Zn-Fe-bLf formulation was thoroughly washed with sterile phosphate-buffered saline (PBS). Further, the obtained $\mathrm{Zn}-\mathrm{Fe}-\mathrm{bLf}$ was coated with a chitosan-nanogel layer $(0.1 \%$ $\mathrm{w} / \mathrm{w}$ ) in the presence of sodium triphosphate added dropwise, while magnetically stirring the solution. Later, the mixture was subjected to overnight magnetic stirring, followed by collection of the nanoformulation by centrifugation and thorough washing with sterile PBS. Zn-Fe-bLf NCs were coated with alginate gel $(0.1 \% \mathrm{w} / \mathrm{w})$ in the presence of calcium chloride $\left(\mathrm{CaCl}_{2}\right)$ solution added dropwise while magnetically stirring the solution. The mixture was then subjected to 6 hours' magnetic stirring, and later Zn-Fe-bLf NCs were separated by centrifugation techniques and washed with sterile PBS. Void NCs lacked the Lf component in them.

Fluorescent $\mathrm{Zn}$-Fe-bLf NCs were fabricated by conjugation of the VivoTag XL fluorophore tag onto the chitosan layer, and stable amide linkages were formed between the succinimidyl ester (present on the fluorophore) and amine group (present on the chitosan layer). Later, fluorescent NCs were subjected to thorough purification by chromatographic purification column, in order to remove unbound fluorophore molecules from the already formed fluorescent NCs. Alginate-coated chitosan nanogel-encapsulated Zn-Fe-bLf NCs were obtained, and lyophilization of samples was carried out and utilized for further research studies. The $\zeta$-potential for the NCs was found to be -1.29 and the size $322 \pm 17 \mathrm{~nm}$, due to the outer coating of alginate, with the help of a Malvern Zetasizer Nano ZS.

\section{Characterization of zinc-doped ferrite particles}

Hysteresis-loop measurement for $\mathrm{Zn}-\mathrm{Fe}$ nanoparticles per unit weight under an applied magnetic field was done using a Quantum Design PPMS AC/DC 1.8-400 K, 0-9 T, 0-3,000 Hz magnetometer at $273 \mathrm{~K}$. A Panalytical X'Pert ${ }^{\circledR}$ Pro MRD XL X-ray diffractometer was used for assessment of spinel $\mathrm{Zn}-\mathrm{Fe}_{3} \mathrm{O}_{4}$ crystallographic structure. Freeze-dried nanoparticle powder was placed in the sample holder, and the top layer was smoothened prior to the scan. The sample holder was fixed into the target slot, and a $z$-axis scan position was manually adjusted while a $0.2 \mu \mathrm{m}$-thick copper attenuator was placed at the X-ray generator end. The scan angle was specified from $25^{\circ}$ to $75^{\circ} 2 \theta \mathrm{X}$-ray beam angle, and the scan parameters as 0.02 -unit step size per 2 seconds. Scan-intensity values were obtained in CSV format and the plot reconstructed using Microsoft Excel software. Size and surface morphology of $\mathrm{Zn}-\mathrm{Fe}_{3} \mathrm{O}_{4}$ nanoparticles were assessed by JEOL JEM-2100 LaB6 transmission electron microscope. Samples were prepared on carbon-coated 400-mesh GSCU400C-50 Formvar copper grids, and standard operating protocol was followed with constant $200 \mathrm{kV}$ high-tension voltage and $110 \mu \mathrm{A}$ beam current. Size and surface morphology of $\mathrm{Zn}-\mathrm{Fe}_{3} \mathrm{O}_{4}$ nanoparticles were reevaluated using a Zeiss Supra ${ }^{\mathrm{TM}}$ 55VP FEG scanning electron microscope with Gemini ${ }^{\circledR}$ column. Imaging samples were prepared on a double-sided carbon tape on a $12 \mathrm{~mm}$-diameter metallic stub sample holder and marked for identification, and standard instrument-operating protocol was followed.

\section{In vitro human tissue-culture analysis for Zn-Fe-bLf NCs in cancer cells Cell-culture conditions}

Human cancer cell culture was carried out as a monolayer culture with Leibovitz media (L-15) without $\mathrm{CO}_{2}$ for triplenegative breast cancer cells (MDA-MB-231), Dulbecco's Modified Eagle's Medium for colonic adenocarcinoma cells (Caco2) and 10\% fetal bovine serum, and penicillin (20 units $/ \mathrm{mL}$ ) and streptomycin $(20 \mathrm{mg} / \mathrm{mL}$ ) (all Thermo Fisher Scientific) antibiotic solution were added as growth supplements. Trypsinization (with $0.25 \%$ trypsin-EDTA for 3-5 minutes) of cancer cells was carried at a cell confluence 
of $80 \%-90 \%$. Cancer cells were cultured at $37^{\circ} \mathrm{C}$ in $25 \mathrm{~cm}^{2}$ and $75 \mathrm{~cm}^{2}$ flasks.

Tumor-cell internalization studies for Zn-Fe-bLf NCs Immunocytochemistry was carried out to determine the cellular internalization efficiency of Zn-Fe-bLf NCs in breast cancer cells. Multiwell chamber slides were utilized to culture the cells (concentration $10^{3}$ cells/well). Treatment concentrations of $125 \mu \mathrm{g} / \mathrm{mL}$ of Zn-Fe-bLf NCs along with an equivalent concentration of void NCs were carried out on the cells for 24 hours. Later, NC treatments were removed and cells rinsed thoroughly with sterile PBS. Freshly prepared 4\% paraformaldehyde was utilized for fixation of cells for about 20 minutes. Rinsing of cell layers was carried out with sterile PBS. Cell permeabilization was carried out for 2 minutes with $0.1 \%$ Triton X. Later, cell layers were rinsed with sterile PBS. Further, an immunoperoxidase kit (Vector Laboratories) was utilized for immunocytochemistry analysis. Firstly, a blocking step was performed for 1 hour with $1 \%$ porcine gelatin. Next, cell layers were rinsed with sterile PBS followed by antibody incubations. Primary antibody incubation was performed with goat anti-bLf (Bethyl Laboratories, Montgomery, TX, USA) with a dilution ratio of 1:200 for 1 hour at $37^{\circ} \mathrm{C}$. Later, the primary antibody incubation was removed and cell layers rinsed with sterile PBS. Secondary antibody incubation was carried out with an antigoat peroxidase HRP-conjugate kit with a dilution ratio of 1:200 for 1 hour at $37^{\circ} \mathrm{C}$. Later, cell layers were thoroughly rinsed with sterile PBS and diaminobenzyl substrate (Sigma-Aldrich) was incubated for 10 minutes. Propidium iodide (1\%) staining of nuclei was performed in the dark. Later, cell layers were rinsed with sterile PBS. Further, mounting of slides was carried out and imaging performed by a Zeiss inverted microscope.

\section{Zn-Fe-bLf NC response for tumor colony-formation efficiency}

Clonogenic tumor-formation assays were performed as per the following protocol. Cancer cells were seeded in a sixwell plate at a concentration of $10^{6}$ and allowed to grow till cells reach confluence. Next, Zn-Fe-bLf NC treatment corresponding to protein concentrations of 400 and $800 \mu \mathrm{g} / \mathrm{mL}$ and void NCs (equivalent to $800 \mu \mathrm{g} / \mathrm{mL}$ ) were incubated with the cells for 24 hours. Later, treatments were removed from cell layers and rinsing of cells with sterile PBS was performed. Next, approximately 300 cells from each well were transferred to $1 \%(\mathrm{w} / \mathrm{v})$ agarose coated six-well plates. Further, tumor cells were allowed to grow for 12 days. Then, colony formation was noted from the second day of cell seeding, and colony growth was monitored. After 12 days of incubation, fixation of colonies was performed with $4 \%$ paraformaldehyde. Later, crystal violet (1\%) staining of colonies was performed. Further, colonies were counted by utilization of a colony-counter number in the control. Void NC- and Zn-Fe-bLf NC treatments and colony-formation efficiency were represented in a histogram.

\section{In vivo nude mice (BALB/c nu/nu) studies to evaluate antitumor efficacy of $\mathrm{Zn}$-Fe- bLf NCs in human xenograft $\mathrm{CIMPI}^{+}$ $\mathrm{CIMP2}^{-} / \mathrm{CIN}^{+}$colonic adenocarcinoma and claudin-low, triple-negative (ER-/PR-/ HER2-) breast cancer model $^{-}$}

In vivo nude mouse studies were performed to assess the anticancer ability in human xenograft $\mathrm{CIMP}^{+} / \mathrm{CIMP}^{-} / \mathrm{CIN}^{+}$ colonic adenocarcinoma and claudin-low, triple-negative $\left(\mathrm{ER}^{-} /\right.$ $\mathrm{PR}^{-} / \mathrm{HER} 2^{-}$) breast cancer models by oral administration of $\mathrm{Zn}$ Fe-bLf NCs in the diet. The study was performed as per Deakin University, Australia (animal ethics committee approval no: A91 - 2010), KU-Warangal, India (IAEC/22/a/UCPSc/ KU/2013), and Postgraduate Institute of Medical Education and Research, India (IAEC no: 47/1999/CPCSEA) animal ethics regulations. A C57 BALB/c nu/nu nude mouse model was employed to develop human xenograft colon (Caco2) and breast (MDA-MB-231) cancer. ${ }^{70}$ An AIN-93G diet (Specialty Feeds, Perth, Australia) mixed with $1.2 \%$ w/w of Zn-Fe-bLf NCs was utilized for oral cancer therapy in the form of pellets. The study was approved by the ethics committee of Deakin University (animal ethics committee approval no: A91 - 2010), PGIMER-Chandigarh (IAEC no: 47/1999/CPCSEA) and KUWarangal (IAEC/22/a/UCPSc/KU/2013/).

AIN-93G includes fat, fiber elements, vitamins, carbohydrates, and protein. The diet is free from casein (milk component), in order to nullify the therapeutic adjuvant effect of casein. The components of AIN-93G are barley, soybean meal, mixed vegetable oils, salt, canola oil, wheat, calcium carbonate, magnesium oxide, dicalcium phosphate, trace minerals, and vitamins.

\section{Human xenograft colon cancer studies}

Eight-week-old athymic mice were subcutaneously injected with the Caco 2 tumor cell line ( $10^{7}$ cells/each mouse) and MDA-MB-231 cells ( $10^{6}$ cells/each mouse). (Colon cancer studies, vehicle-control NC-diet group [ $\mathrm{n}=5]$ and $\mathrm{Zn}$-Fe-bLf NC-diet group [ $\mathrm{n}=5]$; breast cancer studies, vehicle-control NC-diet group [n=5] and Zn-Fe-bLf NC-diet group [n=5]).

Later, after a tumor-volume range of $50-60 \mathrm{~mm}^{3}$ was reached, oral administration of the $\mathrm{NC}$ diet was carried 
out. Routine monitoring of mouse weight, body condition, and behavior was performed. Tumor-volume monitoring was carried out with Vernier calipers and calculated by the formula tumor volume $\left(\mathrm{mm}^{3}\right)=0.5 \times \mathrm{L} \times \mathrm{W}^{2}$, where $\mathrm{L}$ is length (longest tumor dimension) and $\mathrm{W}$ is width (shortest tumor dimension). The study was conducted for 90 days, and later vital organs were collected after the mice were killed. Mouse tissues were processed and stored in appropriate media for future work.

\section{Magnetophotothermal therapy}

In vivo human xenograft colon and breast magnetophotothermal therapy was carried out. The following mouse tumor models were studied: control diet - colon $(n=5)$ and breast $(n=5)$, control diet colon + magnetic scratch + photothermal therapy $(\mathrm{n}=5)$; Zn-Fe-bLf NC diet, colon tumor + magnetic scratch + photothermal therapy $(n=5) ; Z n-F e-b L f$ NC diet, breast tumor + magnetic scratch + photothermal therapy $(n=5)$. Magnetic fields were applied over the tumor area twice a day, photothermal lasers were applied at the tumor site around three times a week, and tumor-mass patterns were examined.

\section{Near-infrared fluorescence-based cancer- imaging strategies to evaluate antitumor efficacy of Zn-Fe-bLf NCs in human xenograft $\mathrm{CIMPI}^{+} / \mathrm{CIMP2}^{-} / \mathrm{CIN}^{+}$colonic adenocarcinoma and claudin-low, triple- negative (ER $\left.{ }^{-} / \mathrm{PR}^{-} / \mathrm{HER} 2^{-}\right)$breast cancer}

NIR-fluorescence imaging was carried out with the IVIS-2 Lumina imaging system. Live mouse NIR-fluorescence imaging was conducted by anesthetizing mice for a short time period. Fluorescent Zn-Fe-bLf NCs were detected by the fluorescent signal of the tag in the wavelength range (675-740 nm), and fluorescent photographs were obtained. The obtained mouse images were analyzed to determine the localization and biodistribution patterns of Zn-Fe-bLf NCs in the body. The fluorescent signal obtained at the tumor site was quantified to assess the tumor-regression trend in terms of average radiant efficiency $\left(\left[\mathrm{p} / \mathrm{s} / \mathrm{cm}^{2} / \mathrm{sr}\right] /\left[\mu \mathrm{W} / \mathrm{cm}^{2}\right]\right)$ and area of fluorescent signal at the tumor site. Furthermore, ex vivo mouse organ-imaging analysis was conducted to assess the biodistribution patterns (in terms of average radiant efficiency) of Zn-Fe-bLf NCs in mouse tissues.

\section{In vivo magnetic resonance imaging and computerized tomography analysis}

Mice were subcutaneously injected with $10^{6} \mathrm{Caco} 2$ cells, and tumors were allowed to develop in mice. Once tumors were developed, commercially available MRI contrast agent (Magnevist) and bLf $(1.6 \mathrm{mg} / \mathrm{mL})$ were injected intratumorally. Another set of mice were fed with Fe-bLf NCs $(1.2 \% \mathrm{w} / \mathrm{w})$, and $\mathrm{Zn}-\mathrm{Fe}-\mathrm{bLf} \mathrm{NCs}(1.2 \% \mathrm{w} / \mathrm{w})$ for 48 hours. Untreated mice were maintained as control. Both the $T_{1}$ and $T_{2}$ MRI signal intensities of Magnevist, bLf, Fe-bLf NCs, Zn-Fe-bLf NCs were measured immediately after injection of the MRI agents using Q-engine (33 mT/m at $125 \mathrm{~T} / \mathrm{m} / \mathrm{s}$ ) from Siemens (1.5 T) with a magnet weight of 3,550 kg and a $10 \mathrm{MHz}$ signal rate with resolution of 100 nanoseconds. The CT scans were obtained using the Somatom CT scanner (Siemens Australia) at the Monash Biomedical Imaging Centre, Melbourne. Mice were anesthetized with isoflurane for these studies.

\section{Estimation of iron, calcium, and zinc}

Serum iron concentrations were determined using a previously published procedure, ${ }^{6}$ whereas serum calcium concentrations were determined using a fura-2-acetoxymethyl ester as previously published. ${ }^{12,21}$ Serum zinc concentration was determined using atomic absorption spectrophotometry. ${ }^{22}$

\section{Statistical analysis}

Statistical analysis was performed with Student's $t$-test (unpaired) using online GraphPad software on the triplicate data generated from individual or triplicate experiments. $P<0.05$ denoted statistical significance, whereas $P<0.01$ denoted results that were highly significant, and $P<0.001$ denoted results that were very highly significant.

\section{Results and discussion}

The studies were performed to delineate the multifunctional anticancer nature of novel Zn-Fe-bLf NCs and also to uncover their multimodular property in terms of real-time cancer imaging and therapy. ${ }^{23,24}$ Earlier studies conducted with Fe-bLf NCs nanotherapeutics showed a multifunctional nature in terms of modulation of critical anticancersignaling nodes, boosting the immunosignaling mechanism by enriching the production of B cells, natural killer cells, and interleukin expression. Furthermore, Fe-bLf and Fe-bLf nanoformulations showed increased cytokine expression, promoting macrophage and phagocytic activity. ${ }^{7,8,25}$ Fe-bLf nanoformulations were noted to play a crucial role in mediating anticancer signaling through the cancer stem cell-signaling mechanism. Experimental investigations were conducted with Fe-bLf in combination with chemotherapeutic regimes, such as Taxol. Recent research findings revealed improvised antitumor ability of Fe-bLf in in vivo colon and breast cancer models. ${ }^{7,8,10}$ Further, recent studies conducted with polymeric 
Fe-bLf NC nanoformulations proved antitumor mechanisms of Fe-bLf in the stem cells. ${ }^{8}$ However, no attempt has been made so far to saturate bLf with $\mathrm{Zn}-\mathrm{Fe}_{3} \mathrm{O}_{4}$. The zinc doping was performed in order to provide a theranostic edge to this conjugate. Earlier studies relating to zinc expression and absorption patterns revealed its role in boosting the anticancer response in correlation with therapeutic regimes. An important micronutrient, zinc also plays a key role in boosting immune responses and helps in fighting against disease-state conditions. ${ }^{13,26}$ In consideration of the importance of zinc in relation to its therapeutic ability, Zn-Fe-bLf NCs were studied to understand their combinatorial anticancer activity. The current work enabled examination of novel multifunctional and multimodular Zn-Fe-bLf NCs for their anticancer ability in in vivo human xenograft $\mathrm{CIMP}^{+} / \mathrm{CIMP}^{-} / \mathrm{CIN}^{+}$colonic adenocarcinoma and claudin-low, triple-negative $\left(\mathrm{ER}^{-} / \mathrm{PR}^{-} /\right.$ HER2 $^{-}$) breast cancer models. Further, the multimodular nature of the nanoformulation was delineated by laser-based NIR imaging, MRI, magnetophotothermal imaging, and positron-emission CT therapeutic imaging.

\section{Synthesis and characterization of superparamagnetic zinc-doped ferrite particles and Zn-Fe-bLf NCs}

Ferrite nanoparticles were synthesized with controlled concentration of zinc $\left(\mathrm{Zn}_{\mathrm{x}} \mathrm{Fe}_{3-\mathrm{x}} \mathrm{O}_{4}\right)$ by chemical coprecipitation followed by hydrothermal treatment. Superparamagnetism was typically displayed with maximum saturation magnetization of zinc-doped ferrite nanoparticles $\left(\mathrm{Zn}_{0.4} \mathrm{Fe}_{2.6} \mathrm{O}_{4}\right)$ at a value of $109.8 \mathrm{emu} / \mathrm{g}$ observed by semiconductor quantum interference-device measurement at $273 \mathrm{~K}$ temperature, which would lead to highly effective $T_{2}$ relaxation MRI contrast (Figure 1A). High-resolution images obtained using transmission electron microscopy revealed a smooth spherical morphology of the synthesized nanoparticles, with an average diameter of $24 \pm 2.8 \mathrm{~nm}$ (Figure 1B). The formation of zinc-doped ferrite nanoparticles was confirmed by assessment of crystallographic orientation X-ray diffractometric analysis using the Panalytical X-pert powder X-ray diffractometer (Figure 1C). The size and surface morphology of the nanoparticles was reconfirmed to be smooth and spherical by scanning electron microscopy (Figure 1D).

\section{In vitro human tissue-culture analysis for $\mathrm{Zn}$-Fe-bLf NCs in cancer cells} Tumor-cell internalization studies for Zn-Fe-bLf NCs Immunocytochemistry-based detection of Fe-bLf was clearly observed, as shown in Figure 1E. The brown color is representative of the specific reaction of $\mathrm{Lf}$ antibodies with Zn-Fe-bLf NCs internalized in the MDA-MB-231 cell line. The observations noted in cellular internalization of $\mathrm{Zn}-\mathrm{Fe}-$ bLf NCs in claudin-low, triple-negative $\left(\mathrm{ER}^{-} / \mathrm{PR}^{-} / \mathrm{HER}^{-}\right)$ breast cancer cells (MDA-MB-231) was performed by immunocytochemistry-based detection, and the results conclusively showed remarkable internalization efficiency of Zn-Fe-bLf NCs within 2 hours compared to the void NCs (Figure 1E).

\section{Zn-Fe-bLf NC response for tumor colony-formation efficiency}

Zn-Fe-bLf NCs exhibited a dose-dependent response in terms of colony-growth inhibition in comparison to void NCs and control (Figure 1F). It was observed that with varying treatment concentrations $(400 \mu \mathrm{g} / \mathrm{mL}$ and $800 \mu \mathrm{g} / \mathrm{mL})$ of Zn-Fe-bLf NCs, colony growth-inhibition efficiency was enhanced (Figure 1G). These observations were in accord with our previous findings relating to remarkable colony growth-inhibition ability of Fe-bLf by modulating crucial cell-signaling pathways, including STAT, VEGF, EGFR, and FGF cell-signaling mechanisms. ${ }^{10,27}$

\section{Evaluation of in vivo antitumor efficacy of Zn-Fe-bLf NCs in human xenograft $\mathrm{CIMPI}^{+} / \mathrm{CIMP2}^{-} / \mathrm{CIN}^{+}$colonic adenocarcinoma and claudin-low, triple- negative (ER-/PR ${ }^{-} / \mathrm{HER}^{-}$) breast cancer}

Studies were conducted to examine the in vivo antitumor efficacy of vehicle-control NCs and Zn-Fe-bLf NCs in human xenograft $\mathrm{CIMP}^{+} / \mathrm{CIMP}^{-} / \mathrm{CIN}^{+}$colonic adenocarcinoma and claudin-low, triple-negative $\left(\mathrm{ER}^{-} / \mathrm{PR}^{-} / \mathrm{HER} 2^{-}\right)$ breast cancer. Routine monitoring of tumor volumes was carried out.

Tumor-volume monitoring in human xenograft colon cancer

The vehicle-control NC-diet group $(n=5)$ showed a tumor volume of $52.28 \pm 11.55 \mathrm{~mm}^{3}$, the control group $(\mathrm{n}=5)$ $93.51 \pm 5.26 \mathrm{~mm}^{3}$, and the Zn-Fe-bLf NC-diet group $(\mathrm{n}=5)$ $0.10 \pm 0.073 \mathrm{~mm}^{3}$. Time-dependent and reduction patterns in tumor volume were noted in $\mathrm{Zn}$-Fe-bLf NC-diet mice when compared to the vehicle-control NC-diet group. The ZnFe-bLf NC-diet treatment was started around day 42, after appropriate tumor size had been reached. Once the mice were fed the Zn-Fe-bLf NC diet, it led to downregulation of tumor volume. The mice were fed the diet continuously 


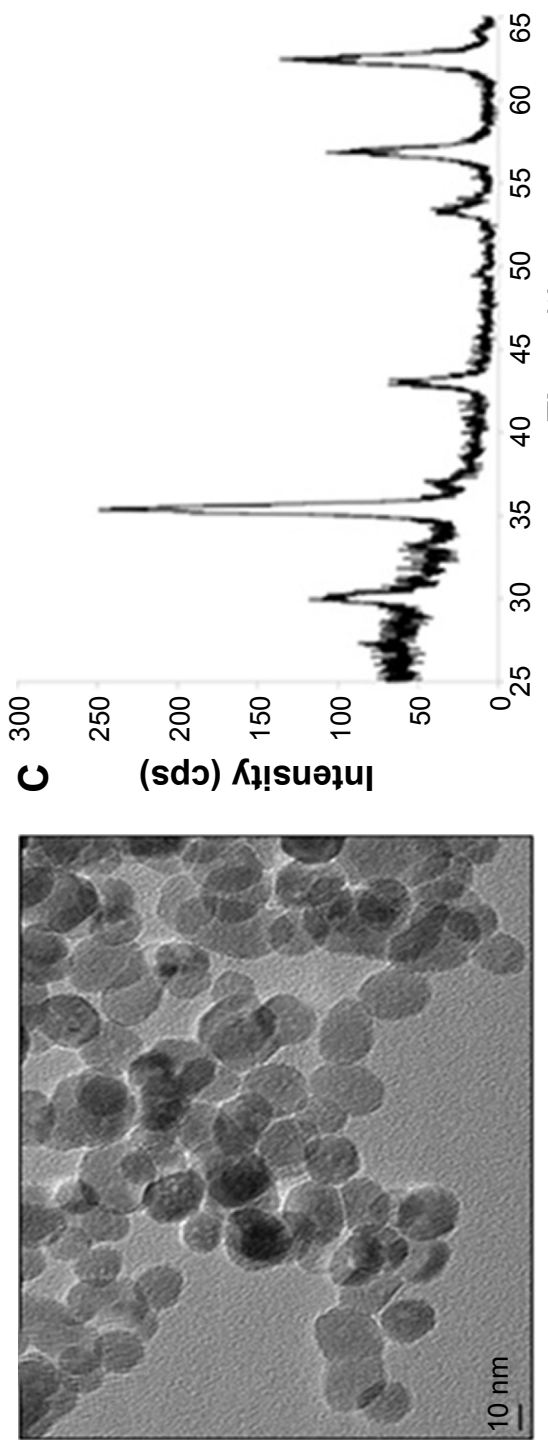

$\boldsymbol{m}$

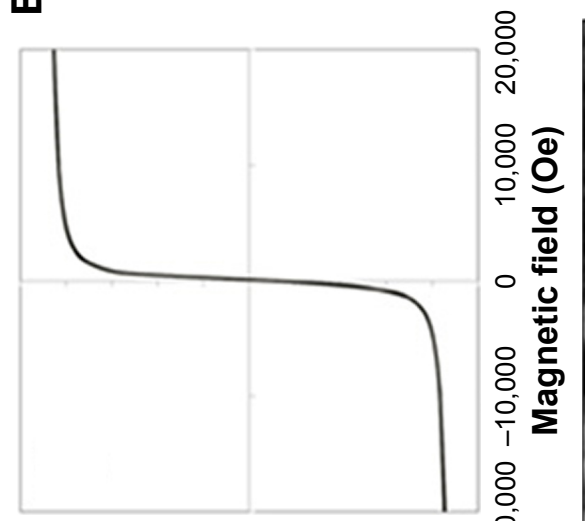

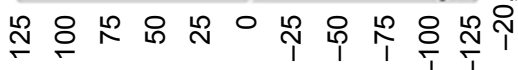

\section{$\varangle$ (6/nшә) uo!ฺez!!әu6ew}
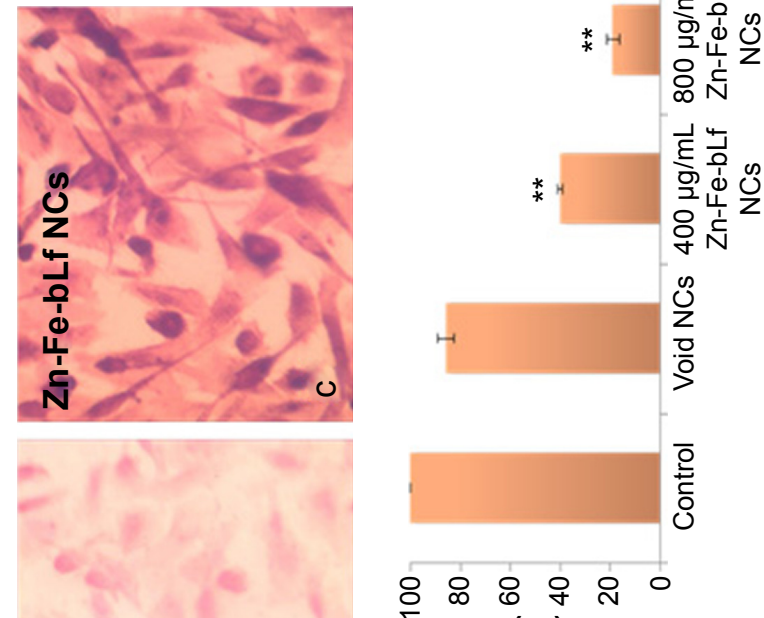

(\%)

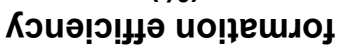

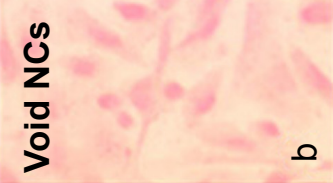

ט -Kuolos

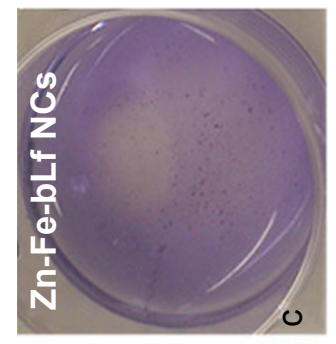

ธ
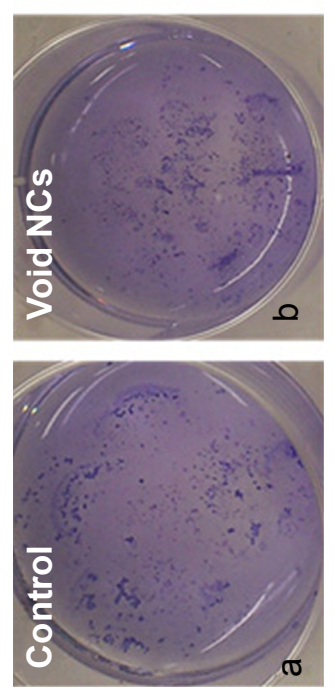

ᄂ

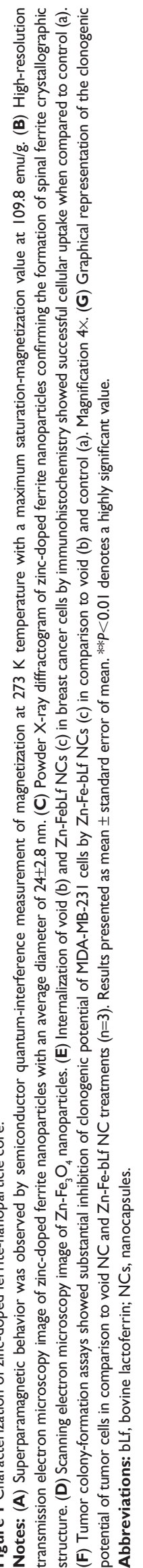


till day 90, and complete regression in tumor volume was observed (Figure 2A). In conclusion, Zn-Fe-bLf NCs showed the potential to be used as an anticancer therapeutic for colon cancer. Collectively, Zn-Fe-bLf NCs exhibited consistent antitumor efficacy and a nontoxic and biocompatible nature in the colon cancer model.
A

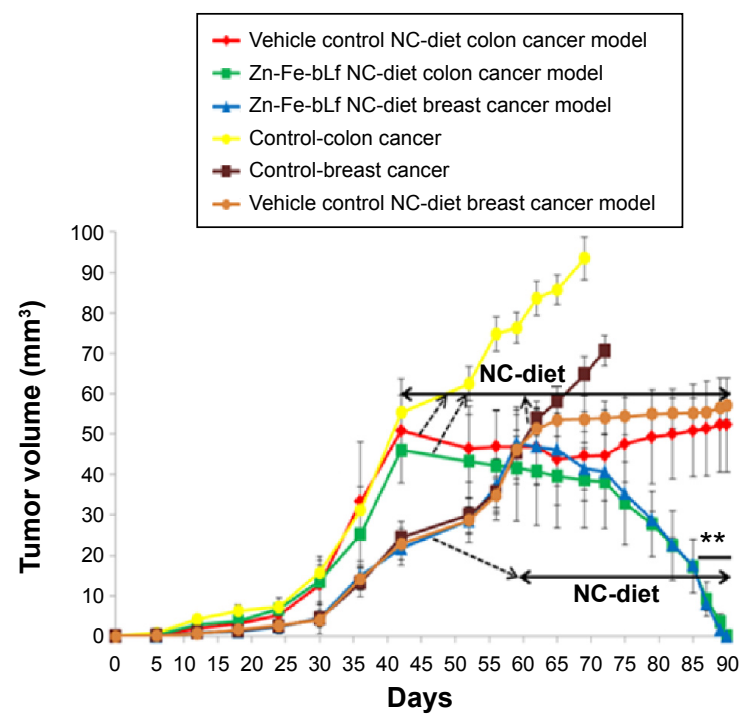

C

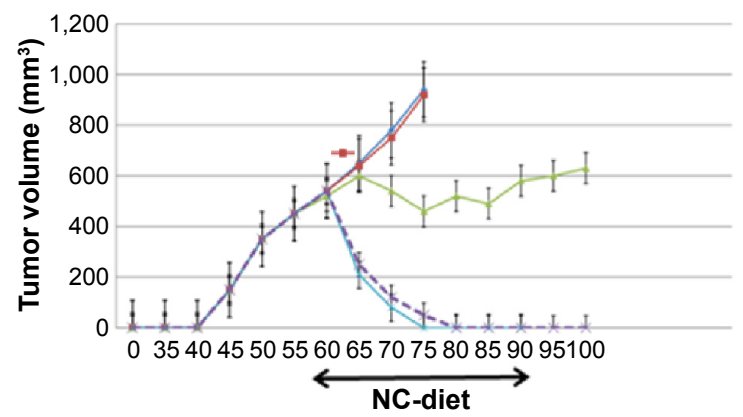

Tumor-volume monitoring in human xenograft breast cancer

The control group ( $\mathrm{n}=5)$ showed a tumor volume of $70.59 \pm 3.75 \mathrm{~mm}^{3}$, the vehicle-control NC-diet group ( $\left.\mathrm{n}=5\right)$ $57.05 \pm 3.33 \mathrm{~mm}^{3}$, and the Zn-Fe-bLf NC-diet group ( $\mathrm{n}=5$ ) $0.051 \pm 0.062 \mathrm{~mm}^{3}$. These observations conclusively establish
B Weight-monitoring chart

- Vehicle control NC-diet colon cancer model

$=$ - Zn-Fe-bLf NC-diet colon cancer model

- Zn-Fe-bLf NC-diet breast cancer model

- Vehicle control NC-diet breast cancer model
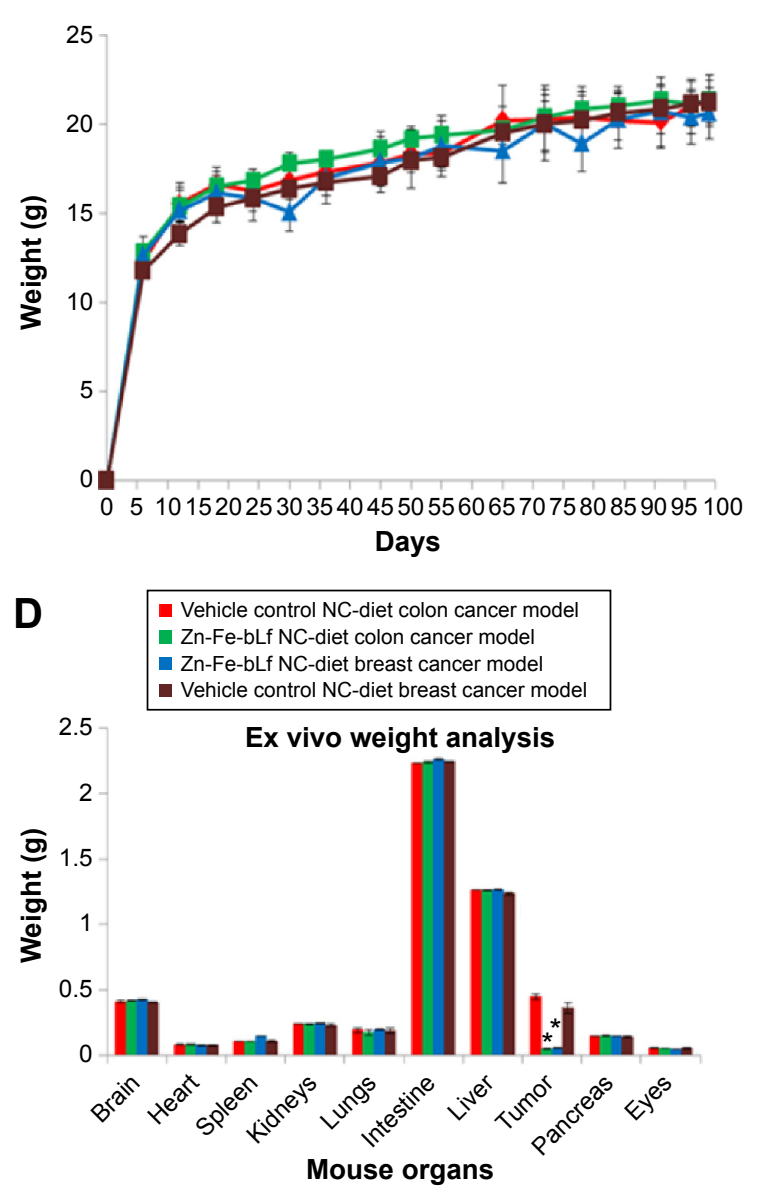

Figure 2 Anticancer efficacy of $\mathrm{Zn}$-Fe-bLf in human xenograft $\mathrm{CIMPI}^{+} / \mathrm{CIMP2}^{-} / \mathrm{CIN}^{+}$colonic adenocarcinoma and claudin-low, triple-negative (ER-/PR-/HER2-) breast cancer model.

Notes: (A) Nanoformulated diet was given to mice at 50-60 $\mathrm{mm}^{3}$ tumor-volume range, around day 42. Colon cancer model: vehicle-control NC-diet group ( $\mathrm{n}=5$ ) showed a tumor-volume of $52.28 \pm 11.55 \mathrm{~mm}^{3}$, with the control group $(n=5) 93.5 \mathrm{I} \pm 5.26 \mathrm{~mm}^{3}$. An increasing trend in tumor volume was observed. The Zn-Fe-bLf NC-diet group ( $\mathrm{n}=5$ ) showed a tumor-volume of $0.10 \pm 0.073 \mathrm{~mm}^{3}$. A regression trend in tumor volume was observed in Zn-Fe-bLf NC-treated mice in comparison to the vehicle-control group. Breast cancer model: tumor volume monitoring was carried out as represented in the chart. The $\mathrm{Zn}$-Fe-bLf nanoformulated diet was given to mice within a $50-60$ mm ${ }^{3}$ tumorvolume range, around day 60 . The $\mathrm{Zn}$-Fe-bLf NC-diet group $(\mathrm{n}=5)$ showed a tumor-volume of $0.05 \mathrm{I} \pm 0.062 \mathrm{~mm}^{3}$, the vehicle-control NC-diet group ( $\left.\mathrm{n}=5\right) 57.05 \pm 3.33 \mathrm{~mm}{ }^{3}$, and the control group $(\mathrm{n}=5) 70.59 \pm 3.75 \mathrm{~mm}^{3}$. A regression trend in tumor volume was observed in Zn-Fe-bLf NC-treated mice. Results presented as mean \pm SEM. (B) Colon cancer: mouse body weight was routinely monitored, as shown in the chart. The vehicle-control NC-diet group ( $\mathrm{n}=5$ ) showed a weight of $2 \mathrm{I} .32 \pm \mathrm{I} .45 \mathrm{~g}$, and the $\mathrm{Zn}$-Fe-bLf NCdiet group $(n=5)$ showed a weight of $21.36 \pm 0.7 \mathrm{I}$ g. A consistent increasing trend in weight was observed. Breast cancer: the $Z n-F e-b L f N C$-diet group ( $n=5)$ showed a weight of $20.64 \pm 1.64 \mathrm{~g}$, with the vehicle-control NC-diet group $(n=5) 21.27 \pm 1.19 \mathrm{~g}$. A consistent increasing trend in weight was observed. Results presented as mean \pm SEM. (C) Zn-FebLf NC-diet administration was carried out around day 60 for colon and breast xenograft tumor mice. An increment in tumor growth was observed in the control diet (colon tumor, breast tumor, and colon tumor + magnetic scratch + photothermal therapy), while the Zn-Fe-bLf NC diet (colon tumor + magnetic scratch + photothermal therapy and breast tumor + magnetic scratch + photothermal therapy) showed enhanced tumor reduction. Results presented as mean \pm SEM. (D) Excised mouse organs were subjected to weight analysis, and all organs listed in the figure showed optimal weight patterns in the vehicle-control NC-diet group $(n=5)$, the $Z n$-Fe-bLf NC-diet colon cancer model ( $n=5)$, and the $\mathrm{Zn}$-Fe-bLf NC-diet breast cancer model $(n=5)$. Results presented as mean $\pm \mathrm{SEM}$. $* P<0.05$ denotes significant value and $* * P<0.01$ denotes a highly significant value. Abbreviations: bLf, bovine lactoferrin; CIMP, CPG-island methylator phenotype; CIN, chromosome instability; NC, nanocapsule; SEM, standard error of mean. 
the remarkable ability of Zn-Fe-bLf NCs in downgrading the tumor. Antitumor-response assessment of the Zn-Fe-bLf NCs in the human xenograft breast cancer model showed remarkable tumor downgrading ability, causing tumor regression (Figure 2A). Oral administration of NCs was started around day 60 and continued till day 90. The ZnFe-bLf NC-treatment group exhibited a tumor volume of $0.051 \pm 0.062 \mathrm{~mm}^{3}$. In summary, these observations showed improved antitumor response of Zn-Fe-bLf NCs. Therefore, $\mathrm{Zn}$-Fe-bLf can be considered a safe multifunctional anticancer agent.

Zn-Fe-bLf NC administration caused antitumor effects, as observed in our earlier findings with respect to Fe-bLf nanoformulations. ${ }^{8,10}$ The vehicle-control diet group (colon cancer) showed a slight reduction of initial tumor growth till day 10 . This may be attributed to the ability of the vehicle control to generate free radicals and reactive oxygen species that caused a temporary slowdown; ${ }^{28}$ however, in the long term an increasing trend in tumor-growth patterns was observed in the vehicle-control diet group. Collectively, these findings reveal in vivo antitumor efficacy in the human xenograft colon and breast cancer models. Interestingly, zinc absorption had a positive correlation with Fe-bLf anticancer function, and findings showed that Fe-bLf exhibited improved antitumor response in correlation with increased expression of intracellular zinc absorption in the blood. Therefore, the novel correlation observed with respect to the zinc and Fe-bLf function might enable the effective functioning of $\mathrm{Zn}-\mathrm{Fe}-\mathrm{bLf}$ $\mathrm{NCs}$ in promoting antitumor actions. Cellular internalization mechanisms of Fe-bLf have been extensively investigated, and are mainly promoted via LRP1 and -2, DMT1, and transferrin receptors. ${ }^{10,24,27}$ Absorption of zinc can also be promoted via zinc-specific receptors ${ }^{29}$ and DMT1. ${ }^{30-32}$ Therefore, understanding the in vivo internalization mechanisms of $\mathrm{Zn}-\mathrm{Fe}-\mathrm{bLf} \mathrm{NCs}$ is a goal for the future.

\section{Evaluation of magnetophotothermal effect of Zn-Fe-bLf NCs in human xenograft $\mathrm{CIMPI}^{+} / \mathrm{CIMP}^{-} / \mathrm{CIN}^{+}$colonic adenocarcinoma and claudin-low, triple- negative (ER $-/ \mathrm{PR}^{-} / \mathrm{HER} 2^{-}$) breast cancer}

Magnetophotothermal studies in the tumor xenografts revealed that the control-diet group in the colon and breast cancer models in the presence of an applied magnetic field and photothermal lasers showed incremental growth patterns in tumor mass. However, the Zn-Fe-bLf NC-diet colon group + an applied magnetic field + photothermal laser showed a tumor-reduction trend. Similarly, the Zn-Fe-bLf-diet breast cancer group + an applied magnetic field + photothermal laser exhibited downregulation of tumor growth (Figure 2B). These observations revealed that the $\mathrm{Zn}$-Fe-bLf NC diet in association with an applied magnetic field and photothermal laser application at the tumor site caused successful cancer-growth inhibition in both the colon and breast cancer xenografts. Although magnetophotothermal studies have been carried out, they have mostly been on in vitro ${ }^{33}$ or intratumoral models. ${ }^{34}$ This is a noninvasive technique for cancer treatment, and the presence of $\mathrm{Zn}-\mathrm{Fe}_{3} \mathrm{O}_{4}$ provides a superior photothermal therapeutic effect. The superior magnetization of zinc-doped $\mathrm{Fe}_{3} \mathrm{O}_{4}$ was found to be the reason for the substantial magnetophotothermal effects observed in the present study. Therefore, these results establish the utilization of $\mathrm{Zn}-\mathrm{Fe}_{3} \mathrm{O}_{4}$ as a multimodular magnetophotothermal nanotheranostic agent.

\section{Evaluation of in vivo nontoxic nature of $\mathrm{Zn}$-Fe-bLf NCs in human xenograft $\mathrm{CIMPI}^{+} / \mathrm{CIMP2}^{-} / \mathrm{CIN}^{+}$colonic} adenocarcinoma and claudin-low, triplenegative (ER ${ }^{-} / \mathrm{PR}^{-} / \mathrm{HER} 2^{-}$) breast cancer

Studies were conducted to evaluate the nontoxic nature of the NCs. The observations included mouse-weight monitoring, body condition and appearance, monitoring water and diet intake, natural behavioral patterns, clinical signatures concerning respiration, and gait movements. Oral administration of the $\mathrm{Zn}$-Fe-bLf NC-formulated diet was carried out with human xenograft colon and breast cancer tumors. Zn-Fe-bLf NCs exhibited a completely nontoxic nature, and mice administered the Zn-Fe-bLf diet showed normal behavioral patterns, normal gait movements, and an optimal weight trend, and in turn mice appeared healthy, showing regular intake of food and water. Oral administration of the nanoformulated diet was carried out in the form of pellets. Weights of the mice were routinely monitored, and a consistent increase in weight was observed in treatment (vehicle-control NC-diet group and $\mathrm{Zn}$-Fe-bLf NC-diet group) models.

\section{Human xenograft colon cancer}

The vehicle-control NC-diet group $(n=5)$ showed weight of $21.32 \pm 1.45 \mathrm{~g}$, the Zn-Fe-bLf NC-diet group $(\mathrm{n}=5)$ $21.36 \pm 0.71 \mathrm{~g}$.

\section{Human xenograft breast cancer}

The vehicle-control NC group exhibited weight of $21.27 \pm 1.19 \mathrm{~g}$ and the Zn-Fe-bLf NC-diet group $(\mathrm{n}=5)$ 
$20.64 \pm 1.46 \mathrm{~g}$. These observations showed a consistent increase in the weight of mice throughout the study period (Figure 2C).

Ex vivo weight analysis was also performed to see the effect of the diets on individual organs, as excessive weight loss has been related to cytotoxicity in organs. ${ }^{35}$ Our results confirmed that no significant differences were observed in weights of individual organs of mice after NC feeding. However, significant weight loss $(P<0.05)$ in tumors in both colon and breast cancer in mice fed $\mathrm{Zn}$-Fe-bLf NC diets were observed when compared to the mice fed the vehicle-control diet (Figure 2D). In summary, the in vivo evaluation of $\mathrm{Zn}-\mathrm{Fe}-\mathrm{bLf}$ in human xenograft colon and breast cancer studies proved to be absolutely nontoxic and highly biocompatible and improved mouse health, giving hope for development of Zn-Fe-bLf NCs as a safe and nontoxic oral anticancer therapeutic.
Near-infrared fluorescence-based

live mouse imaging strategy in human xenograft $\mathrm{CIMPI}^{+} / \mathrm{CIMP}^{-} / \mathrm{CIN}^{+}$colonic adenocarcinoma and claudin-low, triplenegative (ER-/PR-/HER2 ${ }^{-}$) breast cancer

The multimodular nanotheranostic capability of Zn-Fe-bLf NCs was revealed by employing real-time NIR fluorescencebased laser imaging. For imaging studies, the VivoTag 680 XL (fluorochrome dye used for NIR imaging) was encapsulated within the chitosan-sodium triphosphate layer of the NCs. Imaging studies were conducted with the IVIS Lumina NIR fluorescence-imaging system. Selective localization of the Zn-Fe-bLf NCs was observed at the tumor site in the human xenograft colon cancer model (Figure 3A). The biodistribution of NCs was also determined in mice after complete regression of tumors (Figure 3A).
A
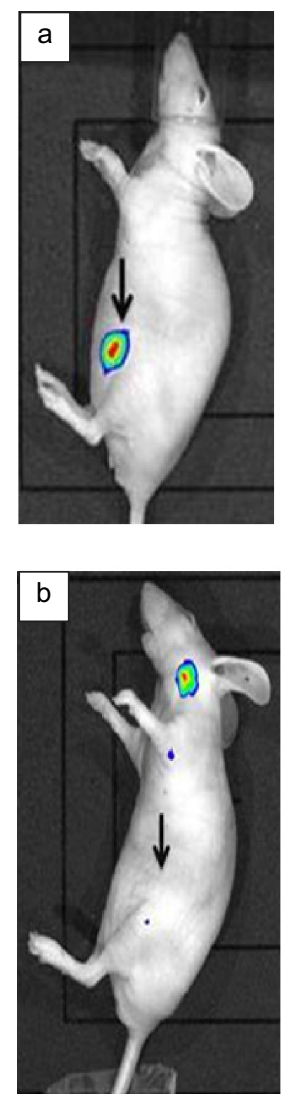

B
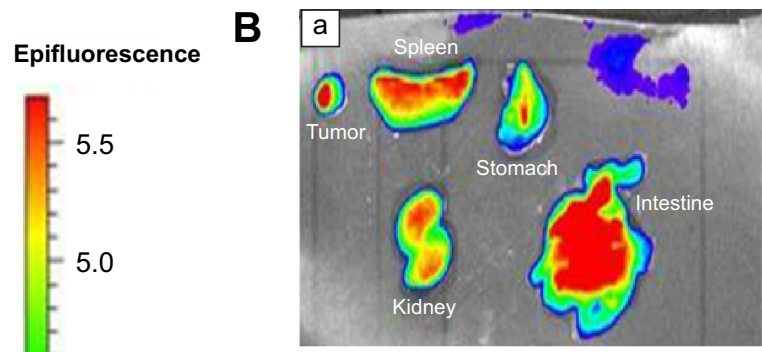

C
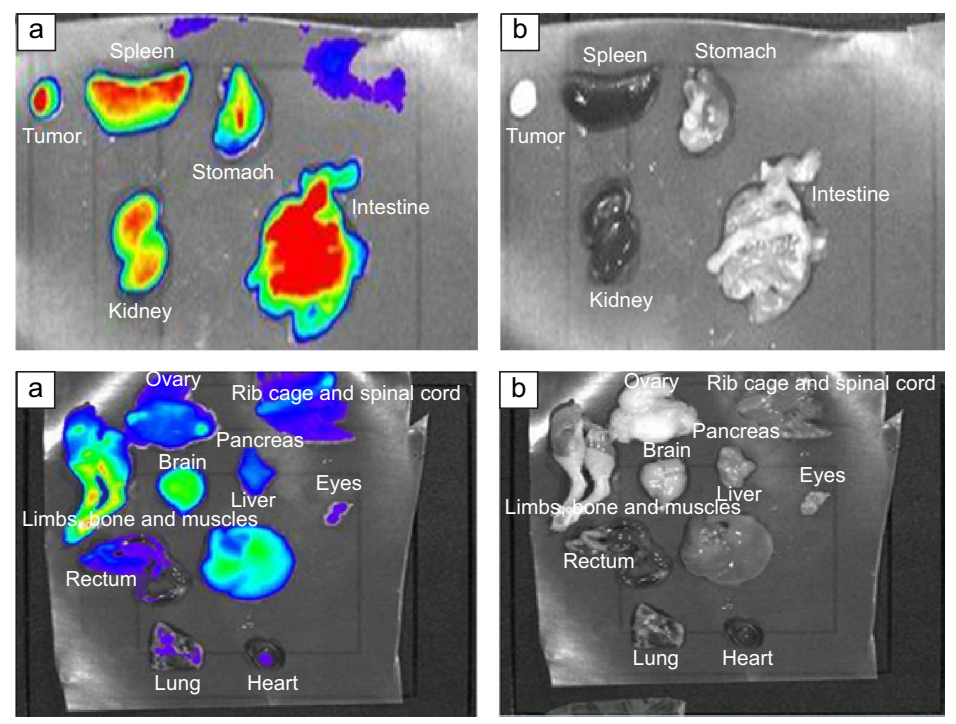

$4.5 \frac{\text { 음 }}{x}$

4.0

$-3.5$

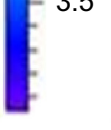

Radiant efficiency $\left(\frac{\mathrm{p} / \mathrm{sec} / \mathrm{cm}^{2} / \mathrm{sr}}{\mu \mathrm{W} / \mathrm{cm}^{2}}\right)$ \begin{tabular}{|l|}
\hline Color scale \\
Min $=3.05^{7}$ \\
Max $=5.7^{7}$ \\
\hline
\end{tabular}

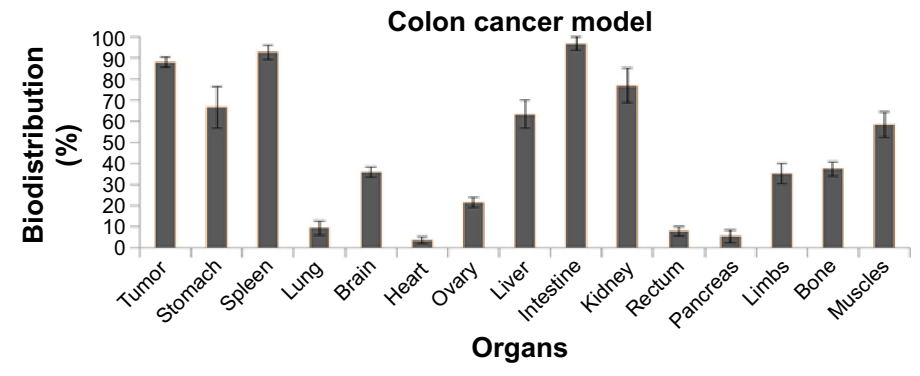

Colon cancer model

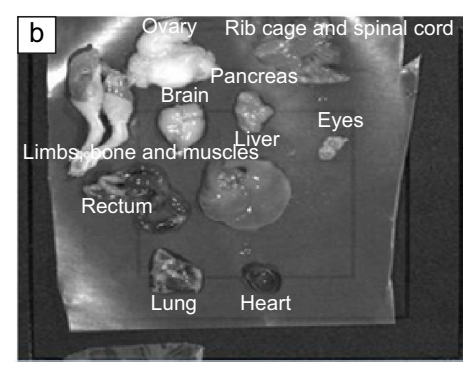

D

Figure 3 Near-infrared fluorescence-based live mouse imaging in human xenograft $\mathrm{CIMPI}^{+} / \mathrm{CIMP2}^{-} / \mathrm{CIN}^{+}$colonic adenocarcinoma model.

Notes: (A) (a) Selective localization of Zn-Fe-bLf NCs at the colon cancer-tumor site. (b) Regression in tumor volume after treatment of mice with Zn-Fe-bLf NCs over time. Arrows demarcate the location of tumour. The Zn-Fe-bLf NC-localization trend was quantified in terms of radiant efficiency to denote the antitumor efficacy of Zn-Fe-bLf NCs in the human xenograft colon cancer model $(n=5)$. (B) (a) Localization of $Z n-F e-b L f ~ N C s$ was noticed in tumors, stomach, spleen, kidney, and intestine. The fluorescence intensity of $\mathrm{Zn}$-Fe-bLf NCs was represented in terms of radiant efficiency. The image is representative of the imaging analysis performed in the Zn-Fe-bLf NC-treatment group ( $\mathrm{n}=5$ ). (b) Ex vivo black-and-white imaging of mouse organs was carried out. The image is representative of the imaging analysis performed in the Zn-Fe-bLf NC-treatment group ( $n=5$ ). (C) (a) Ex vivo fluorescence imaging of mouse organs was carried out. All mouse organs denoted showed sparse localization of Zn-Fe-bLf NCs. The image is representative of the imaging analysis performed in the $\mathrm{Zn}$-Fe-bLf NC-treatment group $(n=5)$. (b) Ex vivo black-and-white imaging analysis of mouse organs. The image is representative of the imaging analysis performed in the Zn-Fe-bLf NC-treatment group ( $n=5$ ). (D) Biodistribution patterns of Zn-Fe-bLf NCs in mouse organs in terms of percentage in vital organs of mice ( $n=5$ ).

Abbreviations: CIMP, CPG-island methylator phenotype; CIN, chromosome instability; bLf, bovine lactoferrin; NCs, nanocapsules. 
Similar observations were made in the xenograft breast cancer model, where maximum localization of Zn-Fe-bLf NCs was found to be present in the tumor (Figure 4A), while in mice with complete regression of tumors the $\mathrm{NCs}$ were located elsewhere (Figure 4A). A time-dependent, consistent decremental pattern of tumor volume was noted, as observed by the in vivo NIR-fluorescence imaging of Zn-Fe-bLf NCs. The obtained images were quantified in terms of radiant efficiency, area $\left(\mathrm{cm}^{2}\right)$, area (in terms of charge-coupled-device camera pixels) for graphical representation of tumor reduction in the mice (Figures S1 and S2). In our previous research, we have not noted any immunostimulatory effects of the vehicle control. ${ }^{8}$ However, in the present study (Figure 2A), it was observed that mice fed on the vehicle-control diet showed relatively low tumor volumes when compared to control mice. This could primarily be due to the anticancer activity of chitosan. This is mainly because the nanoparticles generally accumulate at the tumor site due to the enhanced-permeability-and-retention effect, in order to induce anticancer activity. ${ }^{7}$

Collectively, these observations show the remarkable capability of Zn-Fe-bLf NCs for preferential localization at the tumor site. Zn-Fe-bLf NC localization was monitored over time, and an increase in the theranostic ability for a longer time period was observed. Improved retention of Zn-Fe-bLf NCs was noted, which helped in increased antitumor response in the reticuloendothelial system of the mouse body. The Zn-Fe-bLf NCs were quantified in realms of radiant efficiency and area $\left(\mathrm{cm}^{2}\right.$, charge-coupled device pixels) to denote the time-dependent antitumor response exhibited by Zn-Fe-bLf NCs (Figures S1 and S2). These novel findings reveal interesting observations of Zn-Fe-bLf NC multimodular antitumor activity. In summary, the multimodular imaging capacity of Zn-Fe-bLf NCs revealed tumor-specific localization, promising therapeutic activity as monitored by the NIR-imaging modality. Furthermore, tumor-specific drug targeting with improved therapeutic efficiency can be achieved by employing combinatorial therapeutic imaging platforms, including MRI and positron-emission tomography.

Tumor-specific localization and increased intestinal uptake of Zn-Fe-bLf NCs was significantly mediated via LRP, TfR, and Lf receptors, which are located on their surface, as observed in our earlier findings with Fe-bLf NC nanoformulations. ${ }^{8,10}$ In turn, iron-specific receptors, such as DMT1, ferroportin, and ferritin receptors, can also have a crucial role in absorption of Zn-Fe-bLf NCs. Zn-Fe-bLf $\mathrm{NC}$ retention was detected in the spleen, and could have been due to the presence of iron-specific receptors, splenic macrophage receptors that increasingly promote the uptake of Zn-Fe-bLf NCs. Kidney absorption of Zn-Fe-bLf NCs showed that Zn-Fe-bLf NCs are easily eliminated through the system without causing toxicity in the mouse body. The biodistribution patterns of $\mathrm{Zn}$-Fe-bLf NCs in various mouse organs are denoted in Table 1.

Research has confirmed that zinc-internalization receptors are also expressed in abundance on the tumor surface. ${ }^{31}$ In turn, absorption of Zn-Fe-bLf NCs was observed in such tissues as the intestine and stomach, which obviously stands as a testimonial to effective oral delivery of the nanoformulation in the digestive system. The presence of polymeric coatings, such as a chitosan layering and alginategel encapsulation, around the Zn-Fe-bLf protects it from the acidic and alkaline environmental conditions in the digestive system. ${ }^{7}$ Therefore, increased absorption and improved therapeutic retention capability in the mice body was observed. With respect to the absorption ability of Zn-Fe-bLf-based nanoformulations, these findings were conclusively in accordance with our earlier published findings. ${ }^{7,8}$ Further, uptake of the Zn-Fe-bLf in the kidney was noted, suggesting that residual Zn-Fe-bLf NCs were easily eliminated via the renal system, helping to minimize any side effects. Interestingly, enhanced splenic uptake of the Zn-Fe-bLf NCs was also noted. This observation can be attributed to the presence of zinc-absorption and -internalization receptors and also Lf-internalization receptors on the surface of the spleen. ${ }^{6}$ The therapeutic properties of bLf is a consequence of this absorption phenomenon which further includes, promoting anti-tumor response by increasing splenic-based immune-cell activity via anticancer-phagocyte and antitumor-macrophage activity. ${ }^{6,25}$ Furthermore, scarce biodistribution trends of Zn-Fe-bLf NCs were observed in the brain, eye, skin, rectum, ovary, limbs, heart, lungs, bone, muscles, and liver. In summary, the biodistribution trends observed warrant tumor-specific drug delivery of Zn-Fe-bLf NCs. Zn-Fe-bLf NCs exhibited interesting multifunctional and multimodular ability in terms of exhibiting an anticancer nature and multimodular imaging, enabling monitoring of real-time therapeutic effects through NIR-imaging capability.

\section{In vivo magnetic resonance imaging and computerized tomography analysis}

The images obtained using MRI revealed that although the commercially available MRI contrast agent Magnevist (gadopentetate dimeglumine) gave a bright $\left(T_{1}\right)$ contrast at the tumor, it was also located in other tissues, while bLf was 

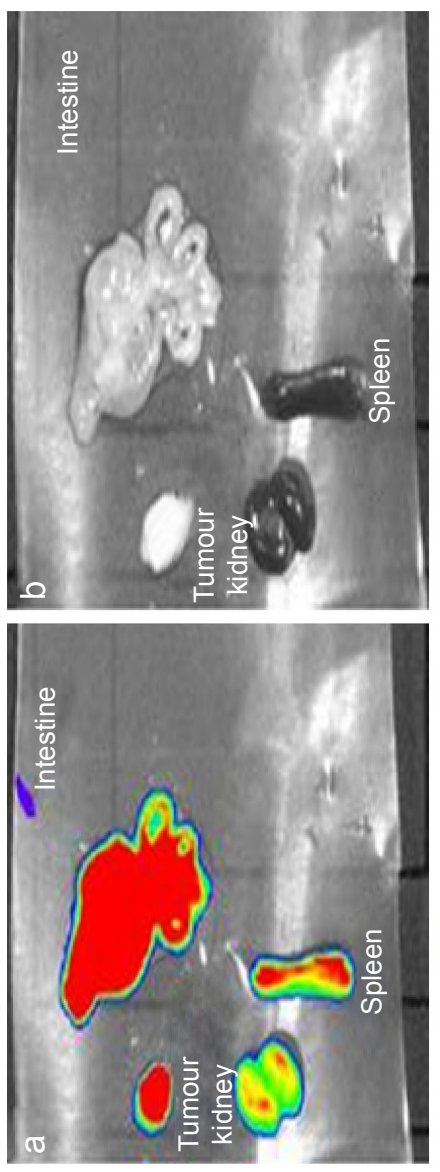

$\mathbf{m}$
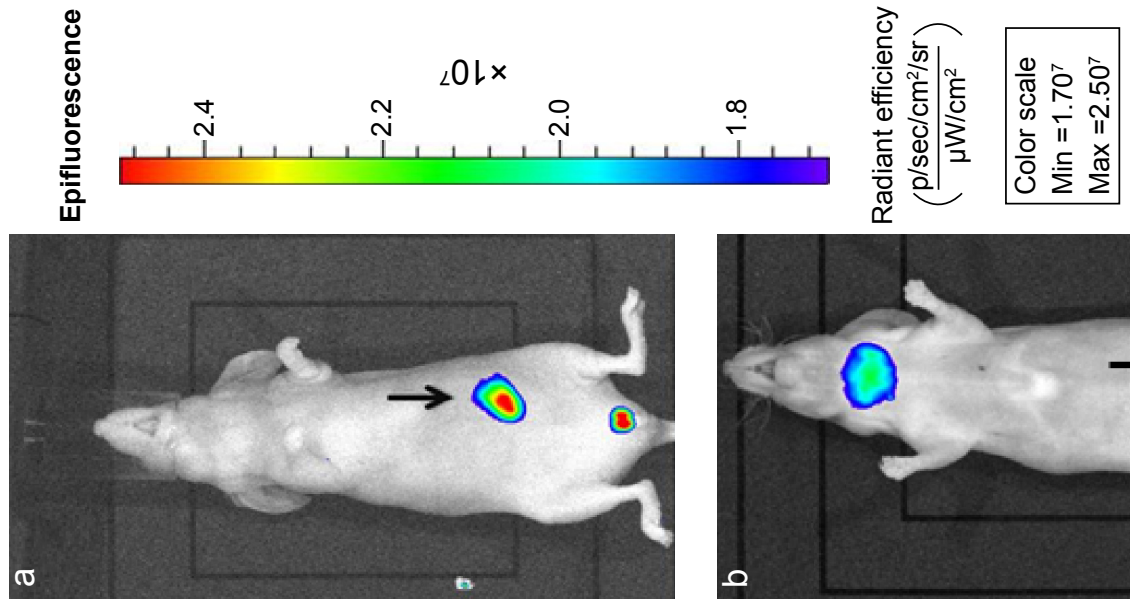
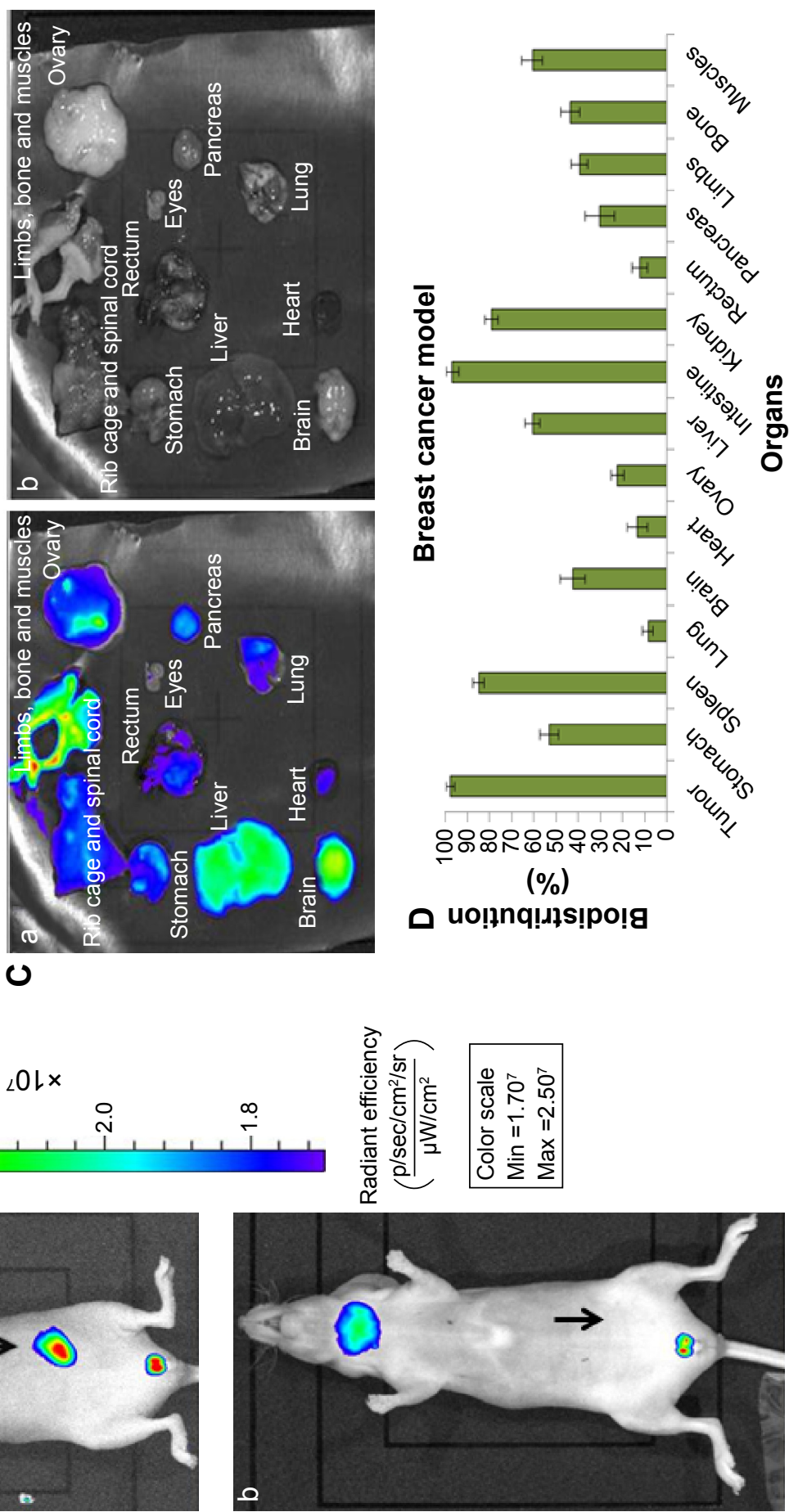

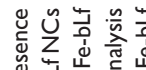

年

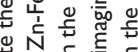

过 $\leftarrow$ 응

要

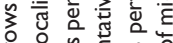

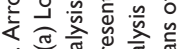

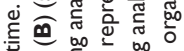

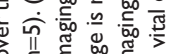

U훰

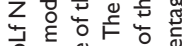

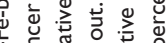

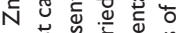

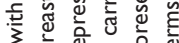

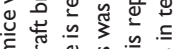

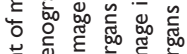

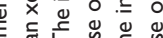

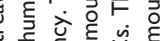

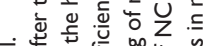

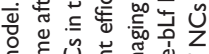

है

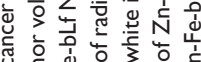

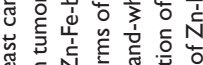

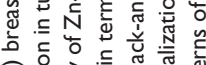

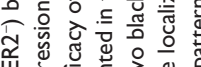

岸

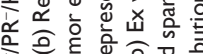

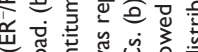

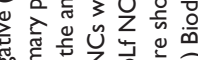

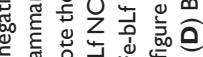

o $\frac{E}{2}$ 这

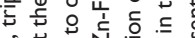

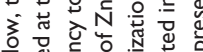

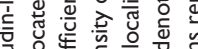

宩 过

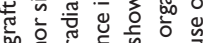

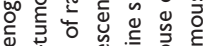

$\times$ 过 हैं

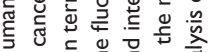

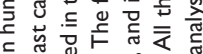

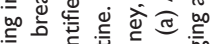

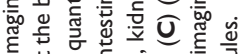

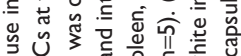

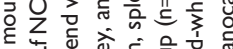

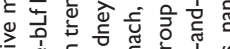

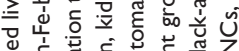

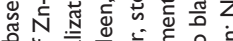

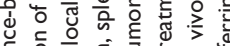

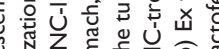

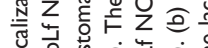

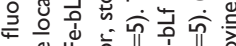

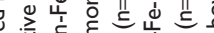

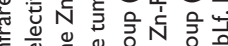

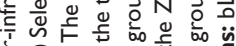

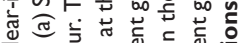

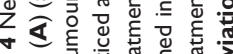

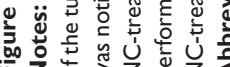


Table I Biodistribution pattern of fluorescent Zn-Fe-bLf NCs in $\mathrm{CIMPI}^{+} / \mathrm{CIMP2}-/ \mathrm{CIN}^{+}$colonic adenocarcinoma and claudin-low, triple-negative (ER-/PR-/HER2 ${ }^{-}$) breast cancer mouse tissues

\begin{tabular}{llll}
\hline Sample & Mouse organ & $\begin{array}{l}\text { Colon cancer } \\
\text { mouse tissues }\end{array}$ & $\begin{array}{l}\text { Breast cancer } \\
\text { mouse tissues }\end{array}$ \\
\hline $\mathrm{I}$ & Brain & $\mathrm{I}+$ & $\mathrm{I}+$ \\
2 & Eyes & - & - \\
3 & Heart & - & $\mathrm{I}+$ \\
4 & Spleen & $3+$ & $2+$ \\
5 & Kidneys & $3+$ & $2+$ \\
6 & Lungs & - & $\mathrm{I}+$ \\
7 & Intestine & $3+$ & $3+$ \\
8 & Liver & $2+$ & $2+$ \\
9 & Tumor & $3+$ & $3+$ \\
10 & Pancreas & - & $\mathrm{I}+$ \\
11 & Ovary & - & $\mathrm{I}+$ \\
12 & Rectum & - & $\mathrm{I}+$ \\
13 & Stomach & $2+$ & $2+$ \\
14 & Spinal cord & - & $\mathrm{I}+$ \\
\hline
\end{tabular}

Notes:-, Nil; I+, low; 2+, medium; 3+, high.

Abbreviations: bLf, bovine lactoferrin; NCs, nanocapsules; CIMP, CpG-island methylator phenotype; $\mathrm{CIN}$, chromosome instability. found to be located only at the tumor site, with considerably bright contrast. Orally fed Fe-bLf NCs and Zn-Fe-bLf NCs gave a dark $\left(T_{2}\right)$ contrast, which could be seen to be present at the tumor site, as well as in the intestine. The Zn-Fe-bLf NCs were found to be dispersed around the tumor as well. A bright $\left(T_{1}\right)$ contrast was also observed in these mice, which could have been due to the presence of zinc. On the other hand, Fe-bLf NCs were highly localized in the tumor, and gave only $T_{2}$ contrast (Figure 5A).

$\mathrm{CT}$ scans revealed a white contrast showing the presence of NCs, mainly in the body organs of the mice. The tumor was also seen clearly, and the contrast of Zn-Fe-bLf NCs was better than the Fe-bLf NCs. A higher intensity of Zn-FebLf NCs was observed in various organs in mice, indicating better uptake and clearly a better signal, suggesting that zinc incorporation may lead to improved signal intensity in imaging by $\mathrm{CT}$ scan (Figure 5B). Computerized tomographic studies conducted on $\mathrm{Zn}-\mathrm{Fe}_{3} \mathrm{O}_{4}$ proved to be an efficient
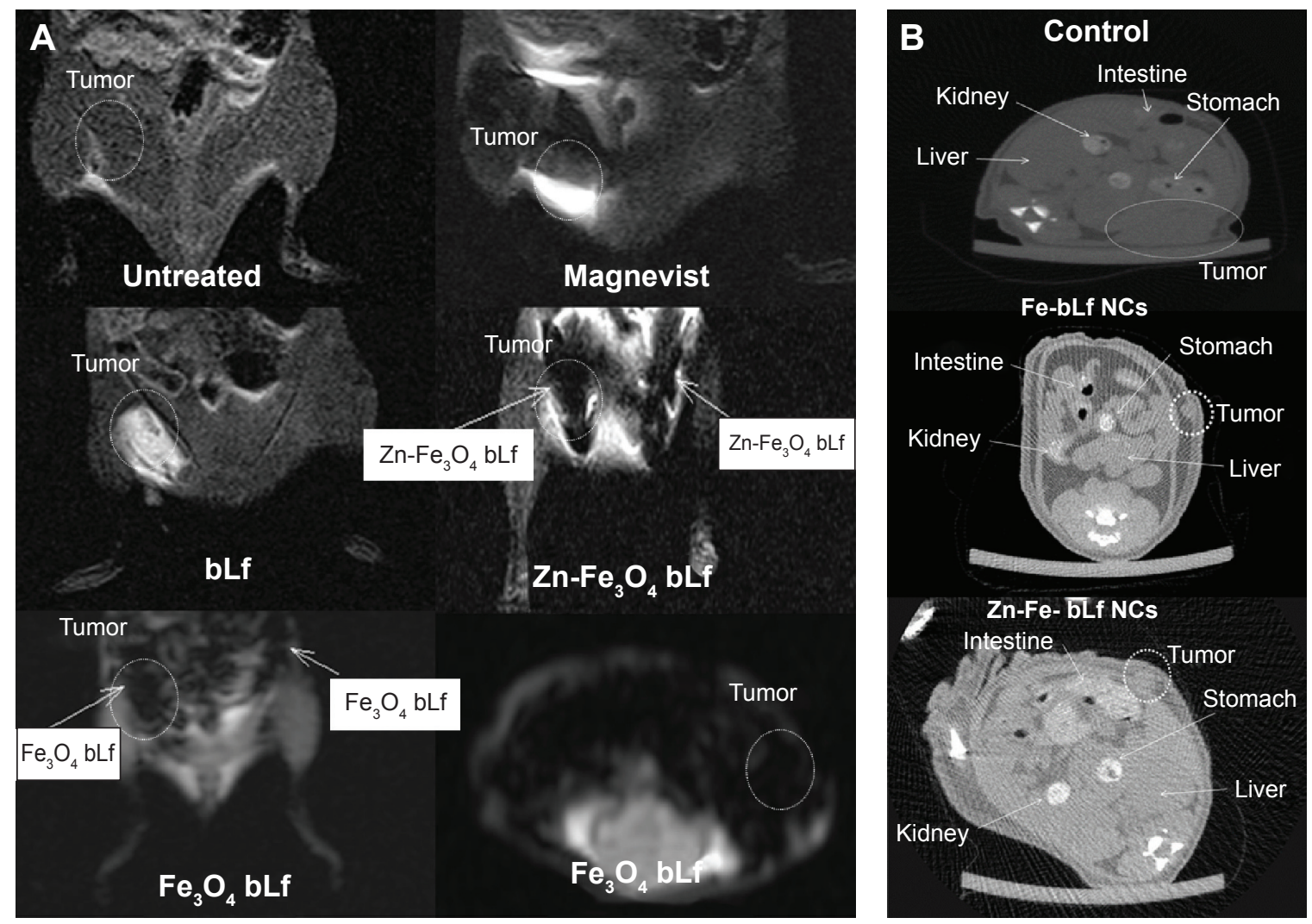

Figure 5 In vivo magnetic resonance imaging and computerized tomography (CT) analysis.

Notes: (A) The upper panel reveals a $T_{1}$ contrast of intratumorally injected Magnevist and bLf. Magnevist was found to move to other tissues apart from the tumor location; however bLf was located in the tumor and gave a better contrast. Both $\mathrm{Fe}_{3} \mathrm{O}_{4}$-saturated bLf NCs and Zn-Fe $\mathrm{O}_{4}$-saturated bLf NCs gave a dark ( $\mathrm{T}_{2}$ ) contrast in the tumor and around the intestines. (B) A bright contrast revealed presence of both $\mathrm{Fe}_{3} \mathrm{O}_{4}$-saturated bLf NCs and $\mathrm{Zn}-\mathrm{Fe}_{3} \mathrm{O}_{4}$-saturated bLf NCs using the CT scans. Zn-Fe $\mathrm{O}_{4}$ saturated bLf was found to be better using CT. The untreated mice served as a control, with little or no contrast.

Abbreviations: bLf, bovine lactoferrin; NCs, nanocapsules. 
Table 2 Serological profiling after Zn-Fe-bLf NC treatment in mice with colon and breast cancer

\begin{tabular}{|c|c|c|c|c|c|c|}
\hline \multirow[t]{2}{*}{ Elemental profiles } & \multicolumn{2}{|l|}{ Control } & \multicolumn{2}{|l|}{ Void NCs } & \multicolumn{2}{|c|}{ Zn-Fe-bLf NCs } \\
\hline & Colon & Breast & Colon & Breast & Colon & Breast \\
\hline Serum calcium $(\mathrm{mg} / \mathrm{dL})$ & $6.36 \pm 2.21$ & $5.7 \pm 1.73$ & $6.85 \pm 1.61$ & $6.63 \pm 2.07$ & $8.38 \pm 0.84$ & $8.03 \pm 1.60$ \\
\hline Serum iron $(\mu g / g)$ & $|43 \pm 2|$ & $138 \pm 16$ & $145 \pm 20$ & $|4| \pm 26$ & $192 \pm 16$ & $187 \pm 19$ \\
\hline Serum zinc $(\mu g / d L)$ & $7.78 \pm 1.48$ & $8.01 \pm 3.15$ & $9.16 \pm 2.11$ & $9.47 \pm 0.89$ & $14.10 \pm 2.83$ & $\mid 4.86 \pm 1.73$ \\
\hline RBC count & $8.70 \pm 1.23$ & $10.03 \pm 1.34$ & $12.62 \pm 1.19$ & $13.53 \pm 2.02$ & $|6.39 \pm 2.7|$ & $18.06 \pm 1.66$ \\
\hline Total hematologic cell count & $9.93 \pm 1.31$ & $8.36 \pm 2.76$ & $10.57 \pm 1.12$ & $\mid 1.03 \pm 3.01$ & $15.26 \pm 2.70$ & $\mid 4.84 \pm 1.74$ \\
\hline
\end{tabular}

Note: Values expressed as mean \pm standard deviation.

Abbreviations: bLf, bovine lactoferrin; NC, nanocapsule; RBC, red blood cell.

nanotheranostic approach in terms of better signal reception and crystal-clear imaging ability. Interestingly, Zn-Fe-bLf NCs proved to be effective multimodular nanotheranostic CT scanning agents, due to their therapeutic properties, and furthermore the observations correlated with earlier studies on $\mathrm{Zn}-\mathrm{Fe}_{3} \mathrm{O}_{4}$ contrast agents.

Blood analysis for detection of calcium, zinc, and iron was performed along with analysis of red blood cell (RBC) and hematologic counts. ${ }^{6,7,35}$ As shown in Table 2, void NCs were able to insignificantly increase serum calcium, iron, and zinc concentrations, along with RBC count and hematologic count. However, Zn-Fe-bLf NCs led to a 1.31-fold (colon) and 1.40fold (breast) increase in serum calcium, while a 1.34-fold (colon) and 1.35-fold (breast) increase in serum iron was observed. The serum zinc level was also observed to increase by 1.81 -fold (colon) and 1.85-fold (breast), while the RBC count increased by 1.88 -fold (colon) and 1.80 -fold (breast) and the total hematologic count was found to increase by 1.53 fold (colon) and 1.77-fold (breast). Maintenance and control of iron homeostasis in cells is essential to prevent deficiency and toxicity of iron. As described in a previous publication, ${ }^{6}$ the domain movements that close or open the iron-binding sites in the $\mathrm{N}$ - and $\mathrm{C}$-lobes regulate the binding and release of iron by bLf. It was also found that a $1.2 \%(\mathrm{w} / \mathrm{w})$ diet of FebLf raised iron levels from $0.0053 \%$ to just $0.0069 \%$, which was statistically insignificant $(P \geq 0.05) .{ }^{6}$ In the present study, the majority of Zn-Fe-bLf was found to localize in the spleen and liver, away from the tumor. Since both these organs are capable of metabolizing the iron, there is no question of increase in metal-ion deposition.

Using pharmacokinetic studies, ${ }^{7}$ we also showed that oral delivery of Fe-bLf ensured presence of Fe-bLf in 3 hours postfeeding, and Fe-bLf was not detectable beyond 72 hours, thus ensuring clearance of protein from the body. Current cancer treatments lead to anemia, due to such side effects as bleeding, hemolysis, deficiency of erythropoietic factors, gut infections, diarrhea, and other nutritional deficiencies. ${ }^{36,37}$ In this study, we have shown clearly that Zn-Fe-bLf NCs substantially increased the serum iron, calcium, and zinc levels, RBC count, and hematologic count. We have already shown that bLf and Fe-bLf have immunomodulatory properties and lead to increase in iron, hemoglobin, and RBC counts. ${ }^{7,25}$ Therefore, $\mathrm{Zn}-\mathrm{Fe}-\mathrm{bLf}$ can also act as an alternative therapy for patients undergoing chemotherapy. ${ }^{38}$

Nude mice were used in the present study, mainly to study the tumor-suppression mechanism of Fe-bLf and not to study the immunomodulatory role. In a previous study, we have shown that Fe-bLf leads to increase in immunomodulatory cytokines. ${ }^{8}$ Therefore, these nanoparticles are capable of targeting cancer cells, as this was a nude mouse model, which is devoid of any immune cells. The anticancer mechanism of Fe-bLf has been provided in previous publications from our laboratory. ${ }^{8,10}$

\section{Conclusion and future directions}

In conclusion, the multifunctional nature of $\mathrm{Zn}-\mathrm{Fe}-\mathrm{bLf} \mathrm{NCs}$ was noted in an in vivo human xenograft $\mathrm{CIMP} 1^{+} / \mathrm{CIMP}^{-}-/$ $\mathrm{CIN}^{+}$colonic adenocarcinoma and claudin-low, triplenegative (ER $\left.{ }^{-} / \mathrm{PR}^{-} / \mathrm{HER} 2^{-}\right)$breast cancer model, leading to preferential downregulation of the tumor mass and also exhibiting safe, eco-friendly characteristics with the nontoxic and highly biocompatible nature of $\mathrm{Zn}-\mathrm{Fe}-\mathrm{bLf}$ NCs. The multimodular activity of the Zn-Fe-bLf NCs was monitored by NIR-fluorescence imaging, revealing a targeted nanotheranostic approach toward colon and breast cancer tumors. Future considerations for work in this area relate to uncover the in vivo anticancer-signaling and stem cell-signaling mechanisms of Zn-Fe-bLf NCs in colon and breast tumors and improvisation of Zn-Fe-bLf NCs for enhancing selective therapeutic effects in combination with imaging strategies, such as ultrasound MRI, single photon-emission CT- and magnetic ultrasound-based imaging modalities. ${ }^{23,39-42}$ Further, new technologies, such as aptamers, locked nucleic acid conjugates, and nanobodies are to be employed in a combinative treatment regime for the safe management of cancer therapies. ${ }^{4,43,44}$ 


\section{Acknowledgments}

The authors would like to thank the Australia-India Strategic Research Fund (AISRF BF030016, BF060042) and the National Health and Medical Research Council (NHMRC APP1050286) for financial support. The authors would like to thank Dr Prasad for the animal work. The authors would also like to thank Dr Nick Branson and Dr Rod Collins from Animal Ethics Committee, Geelong for providing their valuable and necessary help in this study. The authors would also like to thank Dr James Pearson, Dr Qi-Zhu Wu, and Mr Aldo Besmer from the Monash Biomedical Imaging center, Melbourne for their help with the MRI and CT imaging.

\section{Author contributions}

SK performed the in vivo study. KR performed the MRI and CT scans. SK and KR wrote the manuscript. SK, RKK, and JRK had substantial contributions to conception and design, acquisition of data, or analysis and interpretation of data. JRK, RKK, and RS helped in drafting the article or revising it critically for important intellectual content, and arranged funds for this work. RC performed the characterization of nanocarriers and final approval of the version to be published. All authors have agreed on all aspects of the work. All authors contributed toward data analysis, drafting and revising the paper and agree to be accountable for all aspects of the work.

\section{Disclosure}

The authors report no conflicts of interest in this work.

\section{References}

1. Patra HK, Turner AP. The potential legacy of cancer nanotechnology: cellular selection. Trends Biotechnol. 2014;32(1):21-31.

2. Kanwar JR, Mahidhara G, Kanwar RK. Antiangiogenic therapy using nanotechnological-based delivery system. Drug Discov Today. 2011; 16(5-6):188-202.

3. Kanwar JR, Kamalapuram SK, Kanwar RK. Survivin signaling in clinical oncology: a multifaceted dragon. Med Res Rev. 2013;33(4):765-789.

4. Wang Z, Liu G, Zheng H, Chen X. Rigid nanoparticle-based delivery of anti-cancer siRNA: challenges and opportunities. Biotechnol Adv. 2014;32(4):831-843.

5. Wang Z, Niu G, Chen X. Polymeric materials for theranostic applications. Pharm Res. 2014;31(6):1358-1376.

6. Kanwar JR, Palmano KP, Sun X, et al. 'Iron-saturated' lactoferrin is a potent natural adjuvant for augmenting cancer chemotherapy. Immunol Cell Biol. 2008;86(3):277-288.

7. Kanwar JR, Mahidhara G, Kanwar RK. Novel alginate-enclosed chitosan-calcium phosphate-loaded iron-saturated bovine lactoferrin nanocarriers for oral delivery in colon cancer therapy. Nanomedicine (Lond). 2012;7(10):1521-1550.

8. Kanwar JR, Mahidhara G, Roy K, et al. Fe-bLf nanoformulation targets survivin to kill colon cancer stem cells and maintains absorption of iron, calcium and zinc. Nanomedicine (Lond). 2015;10(1):35-55.

9. Kanwar JR, Kamalapuram SK, Kanwar RK. Targeting survivin in cancer: the cell-signalling perspective. Drug Discov Today. 2011; 16(11-12):485-494
10. Mahidhara G, Kanwar RK, Roy K, Kanwar JR. Oral administration of iron-saturated bovine lactoferrin-loaded ceramic nanocapsules for breast cancer therapy and influence on iron and calcium metabolism. Int J Nanomedicine. 2015;10:4081-4098.

11. Patra HK, Khaliq NU, Romu T, et al. MRI-visual order-disorder micellar nanostructures for smart cancer theranostics. Adv Healthc Mater. 2014; 3(4):526-535.

12. Kanwar JR, Kamalapuram SK, Krishnakumar S, Kanwar RK. Multimodal iron oxide (Fe3O4)-saturated lactoferrin nanocapsules as nanotheranostics for real-time imaging and breast cancer therapy of claudin-low, triple-negative (ER-/PR-/HER2-). Nanomedicine (Lond). 2016; 11(3):249-268.

13. US Food and Drug Administration. [homepage on the internet] Guidance for Industry: A Food Labeling Guide (14. Appendix F: Calculate the Percent Daily Value for the Appropriate Nutrients) Available from: http:/www.fda.gov/Food/GuidanceRegulation/GuidanceDocuments RegulatoryInformation/LabelingNutrition/ucm064928.htm. Accessed November 10, 2015.

14. Mekswan K, Sermesri U, Chanvorchote P. Zinc supplementation improves anticancer activity of monocytes in type-2 diabetic patients with metabolic syndrome. Anticancer Res. 2014;34(1):295-299.

15. Tseng YC, Kulp S, Lai IL, et al. Preclinical investigation of the novel histone deacetylase (HDAC) inhibitor AR-42 in the treatment of cancerinduced cachexia. J Natl Cancer Inst. 2015;107(12):274.

16. Shin J, Carr A, Corner GA, et al. The intestinal epithelial cell differentiation marker intestinal alkaline phosphatase (ALPi) is selectively induced by histone deacetylase inhibitors (HDACi) in colon cancer cells in a Kruppel-like factor 5 (KLF5)-dependent manner. J Biol Chem. 2014;289(36):25306-25316.

17. Li H, Chiappinelli KB, Guzzetta AA, et al. Immune regulation by low doses of the DNA methyltransferase inhibitor 5-azacitidine in common human epithelial cancers. Oncotarget. 2014;5(3):587-598.

18. Agarwal VR, Bhatia D, Joshi A, et al. P7170, a novel inhibitor of phosphoinositide 3-kinase (PI3K)-mammalian target of rapamycin (mTOR) and activin receptor-like kinase 1 (ALK1) shows antitumor activity in triple negative breast cancer. Cancer Res. 2014; 74(19 Supplement):4521.

19. Tan Y, Sun D, Jiang W, et al. PP2A-B55 $\beta$ antagonizes cyclin E1 proteolysis and promotes its dysregulation in cancer. Cancer Research. 2014;74(7):2006-2014.

20. Marra P, Mathew S, Grigoriadis A, et al. IL15RA drives antagonistic mechanisms of cancer development and immune control in lymphocyteenriched triple-negative breast cancers. Cancer Res. 2014;74(17): 4908-4921.

21. Kanwar RK, Ganguly NK, Kumar L, Rakesh J, Panigrahi D, Walia BN. Calcium and protein kinase $\mathrm{C}$ play an important role in Campylobacter jejuni-induced changes in $\mathrm{Na}+$ and $\mathrm{Cl}-$ transport in rat ileum in vitro. Biochim Biophys Acta. 1995;1270(2-3):179-192.

22. Chakravarty PK. Evaluation of serum zinc level under malignant condition and its possible implication on improving cell-mediated immunity during cancer progression. World J Oncol. 2011;2(1):16-23.

23. Shah BP, Pasquale N, De G, Tan T, Ma J, Lee KB. Core-shell nanoparticle-based peptide therapeutics and combined hyperthermia for enhanced cancer cell apoptosis. ACS Nano. 2014;8(9):9379-9387.

24. Lee SY, Jeon SI, Jung S, Chung IJ, Ahn CH. Targeted multimodal imaging modalities. Adv Drug Deliv Rev. 2014;76:60-78.

25. Kanwar RK, Kanwar JR. Immunomodulatory lactoferrin in the regulation of apoptosis modulatory proteins in cancer. Protein Pept Lett. 2013;20(4):450-458.

26. Mocchegiani E, Malavolta M, Giacconi R, Costarelli L. Dietary intake and impact of zinc supplementation on the immune functions in elderly: nutrigenomic approach. In: Massoud A, Rezaei N, editors. Immunology of Aging. Heidelberg: Springer; 2014:295-308.

27. Gibbons JA, Kanwar JR, Kanwar RK. Iron-free and iron-saturated bovine lactoferrin inhibit survivin expression and differentially modulate apoptosis in breast cancer. BMC Cancer. 2015; 15:425. doi: 10.1186/s12885-015-1441-4 
28. Laurent S, Saei AA, Behzadi S, Panahifar A, Mahmoudi M. Superparamagnetic iron oxide nanoparticles for delivery of therapeutic agents: opportunities and challenges. Expert Opin Drug Deliv. 2014; 11(9):1449-1470.

29. Imai K, Beppu T, Yamao T, et al. Clinicopathological and prognostic significance of preoperative serum zinc status in patients with hepatocellular carcinoma after initial hepatectomy. Ann Surg Oncol. 2014; 21(12):3817-3826.

30. Degirolamo C, Sabbà C, Moschetta A. Intestinal nuclear receptors in HDL cholesterol metabolism. J Lipid Res. 2015;56(7):1262-1270.

31. Arriaga JM, Greco A, Mordoh J, Bianchini M. Metallothionein 1G and zinc sensitize human colorectal cancer cells to chemotherapy. Mol Cancer Ther. 2014;13(5):1369-1381.

32. Harmaza YM, Slobozhanina EI. [Zinc essentiality and toxicity: biophysical aspects]. Biofizika. 2014;59(2):264-275. Russian.

33. Akhavan O, Meidanchi A, Ghaderi E, Khoei S. Zinc ferrite spinelgraphene in magneto-photothermal therapy of cancer. J Mater Chem B Mater Biol Med. 2014;2(21):3306-3314.

34. Lee HJ, Sanetuntikul J, Choi ES, et al. Photothermal cancer therapy using graphitic carbon-coated magnetic particles prepared by one-pot synthesis. Int J Nanomedicine. 2015;10:271-282.

35. Cox JD, Stetz J, Pajak TF. Toxicity criteria of the Radiation Therapy Oncology Group (RTOG) and the European Organization for Research and Treatment of Cancer (EORTC). Int J Radiat Oncol Biol Phys. 1995; 31(5):1341-1346.
36. Rodgers GM 3rd, Becker PS, Blinder M, et al. Cancer- and chemotherapyinduced anemia. J Natl Compr Canc Netw. 2012;10(5):628-653.

37. Schwartz RN. Anemia in patients with cancer: incidence, causes, impact, management, and use of treatment guidelines and protocols. Am J Health Syst Pharm. 2007;64(3 Suppl 2):S5-S13; quiz S28-S30.

38. Spivak JL, Gascón P, Ludwig H. Anemia management in oncology and hematology. Oncologist. 2009;14 Suppl 1:43-56.

39. Caltagirone C, Falchi AM, Lampis S, et al. Cancer-cell-targeted theranostic cubosomes. Langmuir. 2014;30(21):6228-6236.

40. Conde J, Bao C, Cui D, Baptista PV, Tian F. Antibody-drug gold nanoantennas with Raman spectroscopic fingerprints for in vivo tumour theranostics. J Control Release. 2014;183:87-93.

41. Kanwar RK, Chaudhary R, Tsuzuki T, Kanwar JR. Emerging engineered magnetic nanoparticulate probes for molecular MRI of atherosclerosis: how far have we come? Nanomedicine (Lond). 2012;7(6):899-916.

42. Kiessling F, Fokong S, Bzyl J, Lederle W, Palmowski M, Lammers T. Recent advances in molecular, multimodal and theranostic ultrasound imaging. Adv Drug Deliv Rev. 2014;72:15-27.

43. Leung AW, Kalra J, Santos ND, Bally MB, Anglesio MS. Harnessing the potential of lipid-based nanomedicines for type-specific ovarian cancer treatments. Nanomedicine (Lond). 2014;9(3):501-522.

44. Kanwar JR, Shankaranarayanan JS, Gurudevan S, Kanwar RK. Aptamerbased therapeutics of the past, present and future: from the perspective of eye-related diseases. Drug Discov Today. 2014;19(9):1309-1321. 


\section{Supplementary materials}
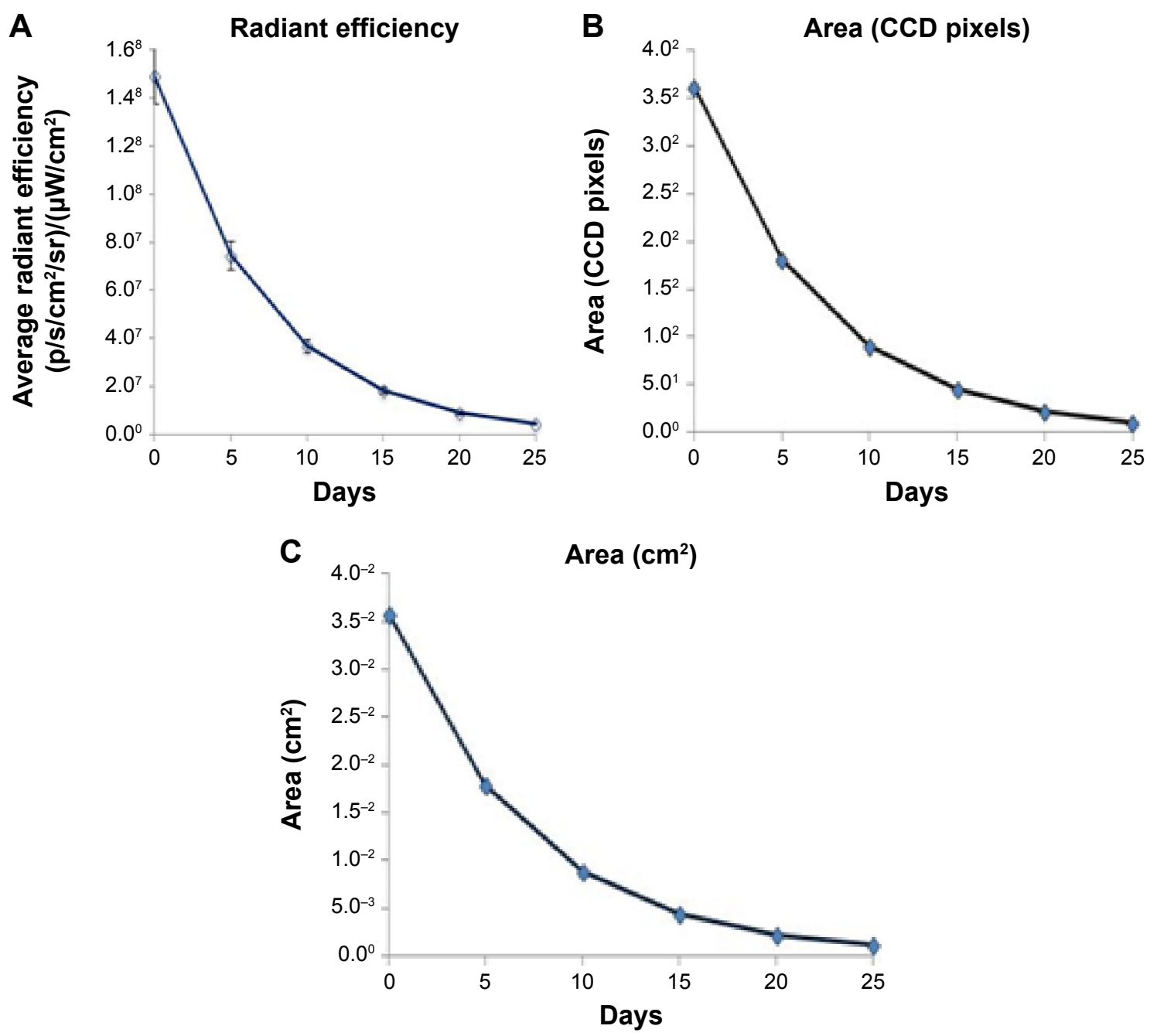

Figure SI Quantification of tumors by radiant efficiency of fluorescent signal in colon cancer model.

Notes: Near-infrared fluorescence imaging showed that Zn-Fe-bLf NCs exhibited a time-dependent downgrading pattern in colon tumor volume. Radiant efficiency of the fluorescent signal at the tumor site, area (in $\mathrm{cm}^{2}$ ) of the fluorescent signal at the tumor site, and area (in terms of CCD pixels) of the fluorescent signal at the tumor site were quantified to denote the decrease in tumor volume over the time period. Radiant efficiency presented as $(\mathbf{A})$ area $\left(\mathrm{cm}^{2}\right)$ and $(\mathbf{B})$ area in terms of CCD pixels. (C) Tumor-reduction trend.

Abbreviations: bLf, bovine lactoferrin; NCs, nanocapsules; CCD, charge-coupled device. 

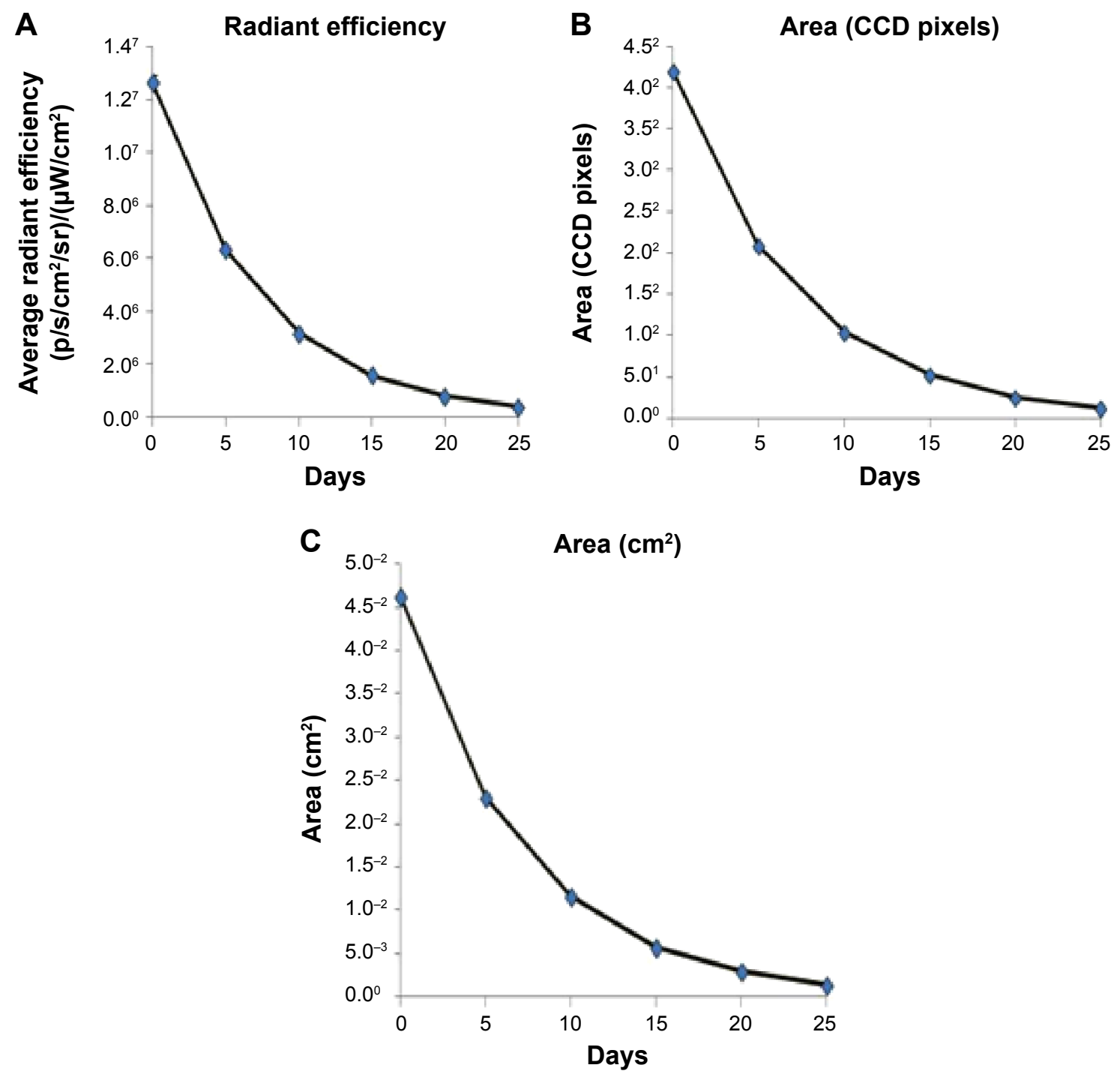

Figure S2 Quantification of tumors by radiant efficiency of fluorescent signal in breast cancer model.

Notes: A time-dependent, consistent decremental pattern in tumor volume was noted, as observed by in vivo near-infrared fluorescence imaging of Zn-Fe-bLf NCs. (A) Radiant efficiency; (B) area in terms of CCD-camera pixels; (C) consistent reduction pattern in tumor volume.

Abbreviations: bLf, bovine lactoferrin; NCs, nanocapsules; CCD, charge-coupled device.

International Journal of Nanomedicine

Dovepress

\section{Publish your work in this journal}

The International Journal of Nanomedicine is an international, peerreviewed journal focusing on the application of nanotechnology in diagnostics, therapeutics, and drug delivery systems throughout the biomedical field. This journal is indexed on PubMed Central, MedLine, CAS, SciSearch ${ }^{\circledR}$, Current Contents ${ }^{\circledR} /$ Clinical Medicine,

Journal Citation Reports/Science Edition, EMBase, Scopus and the Elsevier Bibliographic databases. The manuscript management system is completely online and includes a very quick and fair peer-review system, which is all easy to use. Visit http://www.dovepress.com/ testimonials.php to read real quotes from published authors. 